\title{
PARALLAX OF GALACTIC CEPHEIDS FROM SPATIALLY SCANNING THE WIDE FIELD CAMERA 3 ON THE HUBBLE SPACE TELESCOPE: THE CASE OF SS CANIS MAJORIS
}

\author{
Stefano Casertano ${ }^{1,2}$, Adam G. Riess ${ }^{1,2}$, \\ Jay Anderson $^{1}$, Richard I. Anderson ${ }^{2,3}$, J. Bradley Bowers ${ }^{2}$, Kelsey I. ClubB ${ }^{4}$, Aviv R. Cukierman ${ }^{2,5}$, \\ Alexei V. Filippenko ${ }^{4}$, Melissa L. Graham ${ }^{4}$, John W. MacKenty ${ }^{1}$, Carl Melis ${ }^{6}$, \\ Brad E. TUCKer ${ }^{4,7}$, AND GaUtam Upadhya ${ }^{1,8}$ \\ ${ }^{1}$ Space Telescope Science Institute, 3700 San Martin Drive, Baltimore, MD 21218, USA; stefano@stsci.edu \\ ${ }^{2}$ Department of Physics and Astronomy, Johns Hopkins University, Baltimore, MD 21218, USA \\ ${ }^{3}$ Département d'Astronomie, Université de Genève, Ch. des Mailletes 51, CH-1290 Sauverny, Switzerland \\ ${ }^{4}$ Department of Astronomy, University of California, Berkeley, CA 94720-3411, USA \\ ${ }^{5}$ Department of Physics, Stanford University, Stanford, CA 94305-4060, USA \\ ${ }^{6}$ Center for Astrophysics and Space Sciences, University of California, San Diego, La Jolla, CA 92093-0424, USA \\ ${ }^{7}$ The Research School of Astronomy and Astrophysics, Australian National University, Mount Stromlo Observatory, Canberra, ACT 2611, Australia \\ ${ }^{8}$ Department of Physics, University of Chicago, Chicago, IL 60637, USA \\ Received 2015 December 28; accepted 2016 March 30; published 2016 June 27
}

\begin{abstract}
We present a high-precision measurement of the parallax for the 12-day Cepheid SS Canis Majoris, obtained via spatial scanning with the Wide Field Camera 3 (WFC3) on the Hubble Space Telescope (HST). Spatial scanning enables astrometric measurements with a precision of 20-40 $\mu$ as, an order of magnitude better than pointed observations. SS CMa is the second Cepheid targeted for parallax measurement with $H S T$ and is the first of a sample of 18 long-period ( $\gtrsim 10$ days) Cepheids selected in order to improve the calibration of their periodluminosity relation and eventually permit a determination of the Hubble constant $H_{0}$ to better than $2 \%$. The parallax of SS CMa is found to be $348 \pm 38 \mu$ as, corresponding to a distance of $2.9 \pm 0.3 \mathrm{kpc}$. We also present a refinement of the static geometric distortion of WFC3 obtained using spatial scanning observations of calibration fields, with a typical magnitude $\lesssim 0.01$ pixels on scales of 100 pixels.
\end{abstract}

Key words: cosmology: observations - distance scale - parallaxes - stars: individual (SS CMa) - stars: variables: Cepheids - supernovae: general

\section{INTRODUCTION}

A precise test of the cosmological model can be performed by combining present cosmic microwave background (CMB) measurements (Bennett et al. 2013; Planck Collaboration et al. 2015) with a percent-level determination of the local Hubble constant $H_{0}$ (Hu 2005). More than $70 \mathrm{yr}$ of work, from Hubble (1929) through the first decade of observations with the Hubble Space Telescope (HST), have resulted in a $\sim 10 \%$ measurement of $H_{0}$ (Freedman et al. 2001; Sandage et al. 2006), with much of the remaining uncertainty being of a systematic nature. Riess et al. (2011) sharply reduced the uncertainty to $3.3 \%$, to a value of $73.8 \pm 2.4 \mathrm{~km} \mathrm{~s}^{-1} \mathrm{Mpc}^{-1}$, thanks to four improvements in the distance ladder consisting of Cepheids and Type Ia supernovae (SNe Ia): (1) calibrating eight modern SNe Ia with Cepheids, (2) observing Cepheids in the near-infrared to reduce the impact of extinction and metallicity, (3) using two new geometric calibrations of Cepheids-parallaxes of Galactic Cepheids from the HST Fine Guidance Sensor (FGS; Benedict et al. 2007) and the $3 \%$ geometric maser distance to NGC 4258 (Humphreys et al. 2013, and references therein), and (4) calibrating all extragalactic Cepheid photometry with a single camera, WFC3, to remove cross-instrument zero-point errors.

While local determinations of $H_{0}$ place it in the range of $70-75 \mathrm{~km} \mathrm{~s}^{-1} \mathrm{Mpc}^{-1}$ (see, e.g., the reviews by Livio \& Riess 2013 and Freedman \& Madore 2010), the predictions from CMB measurements with a $\Lambda$ CDM cosmology find a range of 67-70 $\mathrm{km} \mathrm{s}^{-1} \mathrm{Mpc}^{-1}$ (Bennett et al. 2014; Planck Collaboration et al. 2015), indicating tension between the two sets of determinations. A comparative reanalysis of Planck and WMAP data by Addison et al. (2016) (see also Bennett et al. 2013; Planck Collaboration et al. 2015, and references therein) finds that the Planck measurements below $\ell \approx 1000$ are consistent with WMAP, while higher multipoles may be inconsistent. The apparent discrepancy between local measurements of $H_{0}$ and the values predicted from cosmological results may indicate deviation from the $\Lambda$ CDM model or new physics (see, e.g., Wyman et al. 2014), although Bennett et al. (2014) find that the evidence for a discrepancy is inconclusive. A resolution on the origin and magnitude of this potential tension is best found in improving the measurements themselves, especially those at low redshift, which have a larger statistical uncertainty.

Starting with Riess et al. (2009) and then in Riess et al. (2011), we are following a program of rebuilding the foundation of the local distance ladder via an improved calibration of the Cepheid period-luminosity $(P-L)$ relation, also known as the Leavitt Law (Leavitt \& Pickering 1912), to determine accurate distances to nearby hosts of SNe Ia. We have recently started a program to increase the range and precision of trigonometric parallax measurements in the Milky Way in order to reach long-period ( $P>10$ days) Cepheids, nearly all of which are beyond a distance of $2 \mathrm{kpc}$. In Riess et al. (2014, hereafter Paper I) we presented a new observational approach to achieve parallax accuracy of $\sim 30 \mu$ as by spatially scanning the WFC 3 camera on HST. In principle, this method has the promise of achieving a factor of 10-20 improvement over conventional pointed observations or FGS measurements (Benedict et al. 2007; Bellini et al. 2011). This method was demonstrated via five epochs of measurements, spaced every 6 months, of the field around SY Aurigae, a 10- 


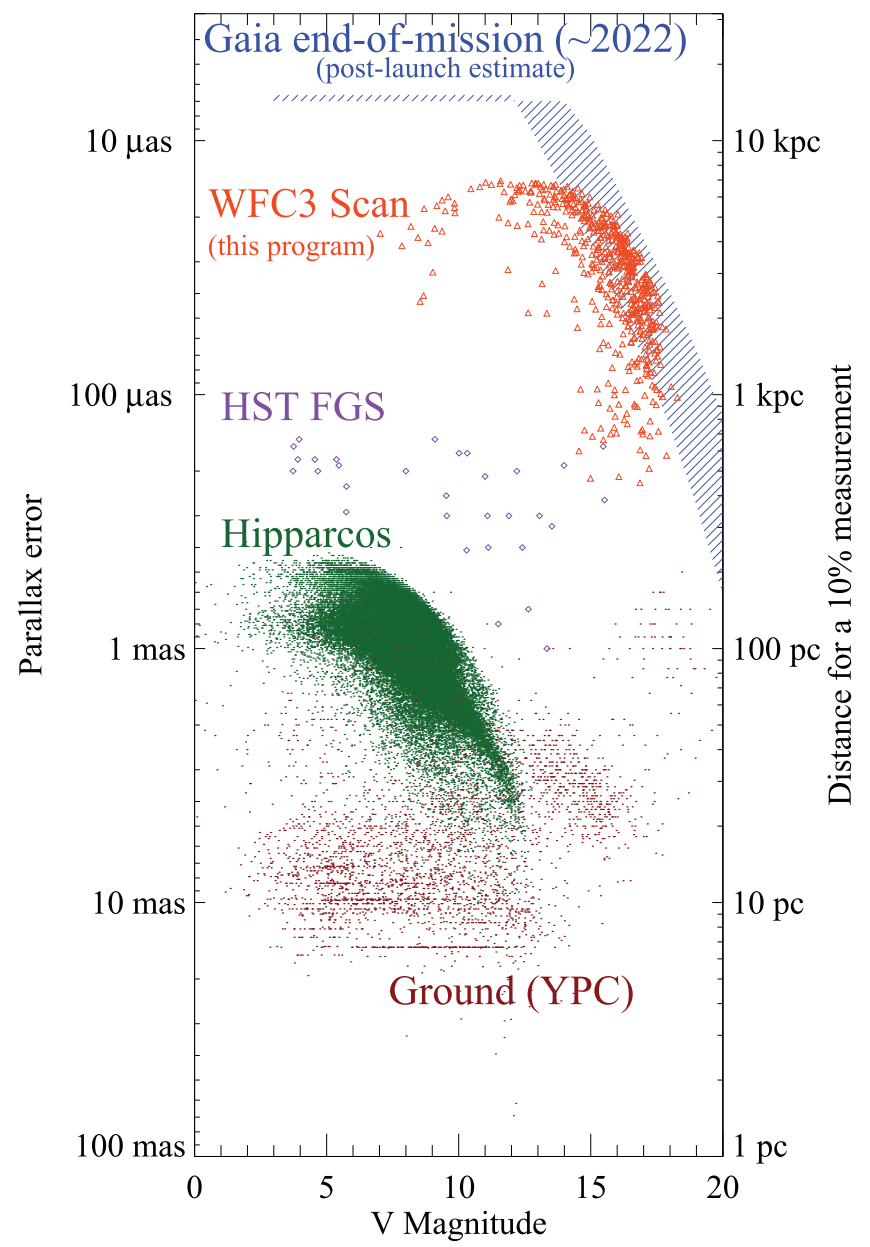

Figure 1. Precision of parallax measurements vs. apparent luminosity from ground and from space, 1995-2022. The right-hand ordinate axis shows the distance at which the error exceeds 10\%. Brown: ground-based measurements from the Yale Parallax Catalog (van Altena et al. 1995). Green: stars with a better than $3 \sigma$ measurement from Hipparcos (Perryman 2009). Purple: measurements based on HST/FGS data (MacConnell et al. 1997; Hershey \& Taff 1998; Benedict et al. 2000, 2001, 2002, 2007, 2009, 2011; Nelan \& Bond 2013). Orange: projected five-epoch precision for target and reference stars from the Cepheid fields observed with HST/WFC3 using spatial scanning. Blue: range of expected precision for Gaia observations, according to the postlaunch estimates in de Bruijne et al. (2015). With the exception of a few radiowavelength measurements (Reid \& Honma 2014), only HST spatial scanning and Gaia can push the $10 \%$ precision horizon beyond $1 \mathrm{kpc}$.

day Cepheid for which we reported a parallax of $428 \pm 54 \mu$ as (statistical). While confirming the promise of the method, Paper I highlighted several improvements in the experiment design necessary to achieve the desired measurement precision of 30-40 $\mu$ as. Most important of these is the selection of targets with a greater number of reference stars in the field, especially those no more than 5 mag fainter than the target Cepheid. We included those considerations in our approved follow-up programs to obtain parallaxes for 18 Galactic Cepheids. The observations of these 18 Cepheids, stretching over five to nine epochs, are now concluding their fifth epoch. Here we present a detailed analysis of the results for the first of these targets, the 12-day Cepheid SS CMa at an expected distance of $\sim 3 \mathrm{kpc}$. Riess et al. (2016) present an updated analysis of the value of $H_{0}$ based on our parallax measurements and the new Cepheids in SN Ia hosts from Hoffmann et al. (2016). The expected precision of the parallax measurement for the target Cepheids and the reference stars in their fields is shown in Figure 1.

In 2013 December, the European Space Agency launched the mission Gaia (Prusti 2012), which promises to determine the fundamental astrometric parameters for $\sim 10^{9}$ stars in the Galaxy with unprecedented precision. Its targets will include hundreds of Galactic Cepheids, including the targets of our $H S T$ program. End-of-mission results from Gaia, expected in 2022, are projected to achieve a parallax precision close to $10 \mu$ as for its bright targets (see Figure 1), although special procedures will be needed for targets brighter than $V \approx 12 \mathrm{mag}$ -including most long-period Cepheids close enough to be effective distance-scale calibrators. Early reports from the mission indicate the existence of significant systematic variations of the basic angle- the separation between the two fields of view $106^{\circ} 5$ apart that lies at the heart of Gaia's ability to measure absolute parallaxes-on periods close to the satellite spin period (Mora et al. 2014). We are optimistic that internal calibrations will enable a full correction for these variations and the eventual achievement of the full expected mission precision shown in Figure 1 (see, e.g., Michalik et al. 2015). Nonetheless, the availability of an external calibration of comparable, if somewhat coarser, precision may also provide a useful verification of the Gaia measurements. Assuming that Gaia achieves its stated goals, the calibration of the $P-L$ relation for Galactic Cepheids will likely be better than $1 \%$ in distance and provide the ideal anchor for a measurement of the local value of $H_{0}$ with unprecedented precision.

The organization of this paper is as follows. In Section 2 we describe the refinements since Paper I in the use of spatial scanning data to measure high-precision, relative astrometry at a single epoch. We also include a description of the calibration observations we have obtained to improve knowledge of the geometric distortion and other instrumental properties of WFC3/UVIS. Section 3 presents the spectroscopic and photometric data we obtained to characterize the properties of the reference stars. We describe in Section 4 refinements in the algorithms used to combine multiple epochs of spatial scan data to measure time-dependent astrometry, and we discuss the parallax measurement thus obtained. In Section 5 we show how radial-velocity information can be used to obtain bounds on the effect that binarity can have on parallax measurements. Section 6 briefly discusses the implications of the present and upcoming measurements.

\section{MW CEPHEID PARALLAXES: A SAMPLE OF 18 TARGETS}

In Paper I we presented our first parallax measurement for a Galactic Cepheid with WFC3 spatial scans, the case of SY Aur. These observations probed for the first time the stability and accuracy of the HST focal plane geometry well below the milliarcsecond (mas) level. Until our scanned observations, the practical limit of relative astrometry with WFC3/UVIS was about 0.01 pixels, or 0.4 mas (Bellini et al. 2011); test data indicated that scanned observations of bright stars over 1000-4000 pixels had the potential to achieve a parallax precision of 20-40 $\mu \mathrm{as}$, about 10 times better than existing measurements. For SY Aur we achieved a final parallax precision of $54 \mu$ as (statistical). However, we were unable to fully determine the systematic uncertainty on this measurement, owing to the paucity of reference stars in its field, which in turn limited our ability to determine the sensitivity of the 
Table 1

Cepheids in Our Sample

\begin{tabular}{|c|c|c|c|c|c|c|c|c|c|}
\hline Name & $\begin{array}{c}\log (P) \\
\text { (days) }\end{array}$ & $\begin{array}{c}\langle B\rangle \\
(\mathrm{mag})\end{array}$ & $\begin{array}{c}\langle V\rangle \\
(\mathrm{mag})\end{array}$ & $\begin{array}{c}\langle I\rangle \\
(\mathrm{mag})\end{array}$ & $\begin{array}{c}\langle J\rangle \\
(\mathrm{mag})\end{array}$ & $\begin{array}{c}\langle H\rangle \\
\langle(\mathrm{mag})\end{array}$ & $\begin{array}{c}\langle K\rangle \\
(\mathrm{mag})\end{array}$ & $\begin{array}{c}\text { R.A. (deg) } \\
(\text { J2000) }\end{array}$ & $\begin{array}{c}\text { Decl. (deg) } \\
(\text { J2000) }\end{array}$ \\
\hline AQ Car & 0.990 & 9.785 & 8.855 & 7.870 & 7.192 & 6.743 & 6.630 & 15.3457 & -61.0741 \\
\hline AQ Pup & 1.479 & 9.70 & 8.54 & 7.175 & 5.879 & 5.329 & 5.091 & 119.5920 & -29.1301 \\
\hline CD Cyg & 1.232 & 10.221 & 8.953 & 7.503 & 6.451 & 5.880 & 5.712 & 301.1107 & +34.1123 \\
\hline DD Cas & 0.992 & 11.111 & 9.880 & 8.580 & 7.552 & 6.952 & 6.908 & 359.3957 & +62.7182 \\
\hline HW Car & 0.964 & 10.122 & 9.125 & 8.027 & 7.258 & 6.704 & 6.596 & 159.8347 & -61.1524 \\
\hline $\mathrm{KN} \mathrm{Cen}{ }^{\mathrm{a}}$ & 1.531 & $\ldots$ & 9.86 & $\ldots$ & 6.27 & 5.92 & $\ldots$ & 204.1537 & -64.5583 \\
\hline RY Sco ${ }^{a}$ & 1.308 & $\cdots$ & 8.19 & $\cdots$ & $\cdots$ & 4.3 & $\cdots$ & 267.7181 & -33.7057 \\
\hline $\mathrm{S} \mathrm{Vul}{ }^{\mathrm{a}}$ & 1.836 & $\ldots$ & 9.17 & $\ldots$ & 5.32 & 4.92 & $\ldots$ & 297.0992 & +27.2865 \\
\hline SS CMa & 1.092 & 11.136 & 9.925 & 8.470 & 7.434 & 6.849 & 6.677 & 111.5300 & -25.2574 \\
\hline SY Aur & 1.006 & 10.071 & 9.066 & 7.854 & 6.899 & 6.399 & 6.391 & 78.1634 & +42.8318 \\
\hline SZ Cyg & 1.179 & 10.909 & 9.430 & 7.797 & 6.573 & 5.886 & 5.746 & 308.2262 & +46.6013 \\
\hline VX Per & 1.037 & 10.459 & 9.307 & 7.995 & 7.076 & 6.517 & 6.292 & 31.9500 & +58.4433 \\
\hline VY Car & 1.277 & 8.616 & 7.455 & 6.279 & 5.463 & 4.944 & 4.804 & 161.1362 & -57.5654 \\
\hline WZ Sgr & 1.339 & 9.400 & 8.017 & 6.530 & 5.402 & 4.763 & 4.565 & 274.2488 & -19.0758 \\
\hline X Pup & 1.414 & 9.742 & 8.515 & 7.157 & 6.180 & 5.600 & 5.430 & 113.2072 & -20.9056 \\
\hline XY Car & 1.094 & 10.510 & 9.294 & 7.950 & 6.978 & 6.405 & 6.240 & 165.5669 & -64.2629 \\
\hline XZ Car & 1.221 & 9.861 & 8.604 & 7.251 & 6.313 & 5.745 & 5.585 & 166.0561 & -60.9799 \\
\hline YZ Car & 1.259 & 9.829 & 8.709 & 7.444 & 6.492 & 5.971 & 5.808 & 157.0702 & -59.3502 \\
\hline $\mathrm{Z} \mathrm{Sct}$ & 1.111 & 10.914 & 9.585 & 8.098 & 7.042 & 6.491 & 6.429 & 280.7386 & -5.8209 \\
\hline
\end{tabular}

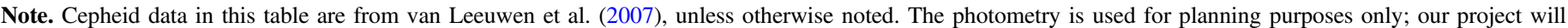
obtain HST photometry for the target Cepheids.

a Data for this object have been obtained from the SIMBAD database (Wenger et al. 2000).

result to different processing choices. In many ways, SY Aur was a test case, and the strict requirements of our measurement process were not known at the start of our first 2 yr campaign.

On the basis of the analysis of the SY Aur results, we have selected a sample of 18 additional Galactic Cepheids for which we could expect to obtain parallax measurements with uncertainty $\sigma=30-40 \mu$ as in order to improve the calibration of the Cepheid $P-L$ relation for the determination of the Hubble constant. Cepheids in this sample are listed in Table 1, together with the basic properties used in their selection; the magnitudes in the table are those reported by van Leeuwen et al. (2007), unless otherwise noted. As part of our program, we will also obtain HST photometry of the target Cepheids in the same filters used for those in SN Ia host galaxies, in order to remove any uncertainties related to differences between ground-based and HST photometric systems. The primary considerations in their selection are (1) period longer than $\sim 10$ days, (2) 10 reference stars within the field (scan length $>500$ pixels) within 5-6 mag of the Cepheid itself, and (3) an estimated distance less than $4 \mathrm{kpc}$ at the $3 \sigma$ level.

The requirement for a period longer than 10 days stems from the desire to minimize the impact of systematic uncertainties when using the $P-L$ relation to measure the Hubble constant. Cepheids in external galaxies, especially the hosts of SNe Ia, can be observed with adequate accuracy only if they are sufficiently bright, which implies longer periods. In practice, most of the information comes from Cepheids with periods longer than 10 days. Calibrating the $P-L$ relation with Cepheids of significantly shorter period introduces a systematic uncertainty related to the slope of the relationship. In addition, there are indications that the $P-L$ relation has a break in the neighborhood of 10 days, represented by a change in its slope (see, e.g., Sandage et al. 2004; Ngeow et al. 2009; Kodric et al. 2015, and references therein). If true, this enhances the reason to use as local calibrators primarily longer-period Cepheids, which follow the same $P-L$ relation as the Cepheids in SN Ia host galaxies. Figure 2 shows the $P-L$ relation for Galactic Cepheids with measured parallaxes and the distribution of periods for the Cepheids in our sample and SN Ia host galaxies (Hoffmann et al. 2016). See also Riess et al. (2016) for an updated analysis of the value of $H_{0}$ based on our new measurements.

As shown by our experience with SY Aur, bright reference stars are critical to constrain the relative orientation and variable geometric transformation between scanning-mode exposures. Shallow exposures - typically either in narrowband filters or with the telescope moving faster than $1^{\prime \prime} \mathrm{s}^{-1}$-are needed to observe the $V \approx 9$ mag Cepheid without saturation. Deep exposures in a broadband filter are needed in order to measure enough reference stars $(V<17 \mathrm{mag})$ to provide a well-constrained absolute parallax, as discussed in Section 4. Shallow and deep scanning observations are obtained within the same orbit, but the analysis of SY Aur data shows that the geometric distortion varies significantly within a single orbit, owing to the HST day-night cycle. Therefore, it is necessary to account for this change in the astrometric registration of images taken within the same orbit; empirically, a second-order polynomial term is sufficient. Unless at least 10 stars are available to determine the second-order polynomial correction, the uncertainty from the correction dominates the uncertainty in the Cepheid measurement. Therefore, we require that at least 10 stars be observable in the shallow exposures with signal-tonoise ratio $>30$ per pixel, which implies stars no more than 6 mag fainter than the Cepheid and a scan length of at least 500 pixels.

Finally, the requirement on estimated distance ensures that a nominal error of $\sim 30 \mu$ as in parallax translates into a $\sim 10 \%$ distance error for each target. Assuming that each Cepheid is at a distance consistent with the current $P-L$ calibration, a final parallax error of $30 \mu$ as for each Cepheid, combined with adequate photometry, would result in a collective calibration of the $P-L$ relation to approximately $0.04 \mathrm{mag}$, or $2 \%$ in distance, 


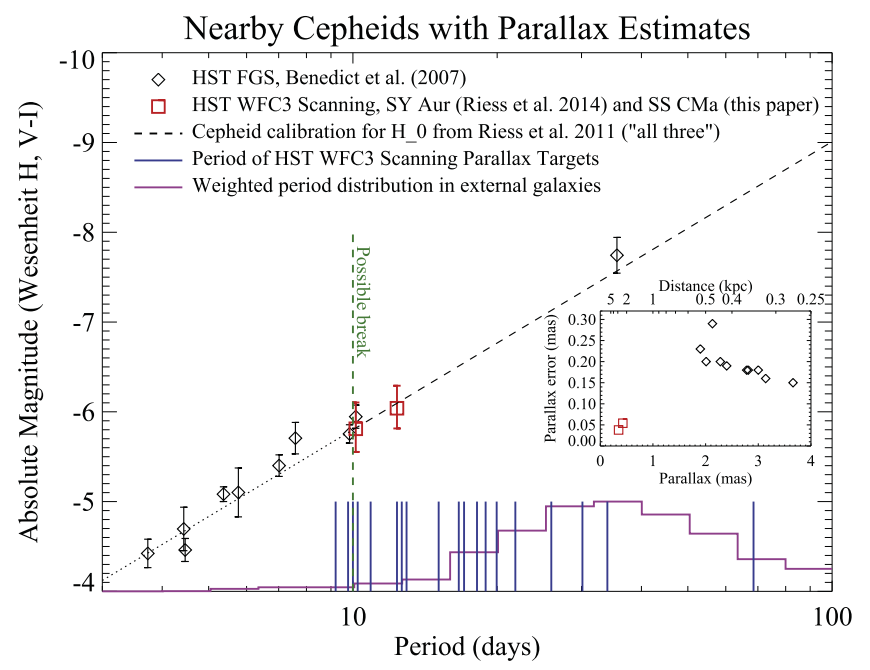

Figure 2. $P-L$ relation for Galactic Cepheids with trigonometric distance measurement, and period distribution for Cepheids used in the $H_{0}$ distance calibration. The open diamonds are the Galactic Cepheids with HST-FGS parallax measurements from Benedict et al. (2007), with the Wesenheit absolute magnitude in the $H$ band estimated from their distance and uncertainty. The red squares are SY Aur and SS CMa, with the distances determined in Paper I and in this paper, respectively. The dotted/dashed line shows the $P-L$ calibration obtained in Riess et al. (2011) when using all three anchors (Galactic Cepheids, LMC, and NGC 4258). Because of a possible break at $P \approx 10$ days (marked by a vertical green line; Sandage et al. 2004 Ngeow et al. 2009; Kodric et al. 2015, and references therein), the line is shown dotted below 10 days and dashed above. The magenta histogram indicates the distribution of periods for Cepheids in SN Ia hosts (Hoffmann et al. 2016; Riess et al. 2016), scaled to the same total weight for each host. The vertical blue bars show the period of all Cepheids in our sample (Table 1). Finally, the inset shows the parallax and error for the Benedict et al. (2007) targets (black diamonds) vs. SY Aur and SS CMa (red squares); note that the absolute parallax error for SY Aur and SS CMa is much smaller than for the previous targets, but the fractional parallax error - and thus the quality of the luminosity calibration-is comparable.

a significant improvement over the $3 \%$ uncertainty of the NGC 4258 calibration (Humphreys et al. 2013).

\subsection{HST Observations}

For each Cepheid, we obtain HST observations in five to nine epochs at 6-month intervals, ensuring that the observations are always executed at orientations $180^{\circ}$ apart to within the $H S T$ pointing precision (about $0^{\circ} .01$ ). The reason is that our measurements are inherently one-dimensional; we obtain very accurate positions perpendicular to the scanning direction, and much less accurate (often less so than direct observations) in the direction along the scan. In order to optimally measure the variation in position of the Cepheid, we need to ensure that the direction of resolution is always nearly the same.

To the extent possible, we also need the scan direction to be fixed with respect to the detector frame and close to the detector $Y$ direction. This minimizes the impact of low-level geometric distortion, for which only the $X$ component is needed, and of the charge transfer efficiency (CTE) effects that are well documented with space-based charge-coupled devices (CCDs; see, e.g., Anderson \& Bedin 2010). In particular, the CTE losses are much smaller in the $X$ than in the $Y$ direction (Anderson 2014a); consequently, it is desirable for the measurement direction to nearly coincide with the detector $X$ direction, implying a scan along $Y$. (A small angular offset is introduced in order to vary the pixel phase along the scan.) The motion of the Cepheid in the resolution direction is the result of the combination of the appropriate component of the proper motion and of the parallax of the target, compared to that of the reference stars. Ideally, the date and orientation of each observation should be chosen to maximize the projection of the parallactic motion along the resolution direction. Because of the $180^{\circ}$ change requirement, the date and allowed orientation range of each observation are constrained, typically resulting in a projection factor of $0.8-0.9$. We allow for a slack of up to 1 week in the scheduling of each observation.

At each epoch, we obtain four or five scanned observations. The first four are straight scans in the sequence: Forward, deep; Backward, shallow; Forward, shallow; Backward, deep. This sequencing helps average out time variations between deep and shallow scans, which could otherwise lead to larger systematic differences between deep scans (for most reference stars) and shallow scans (for the Cepheid and the brighter reference stars).

If possible, a fifth scan is obtained in so-called "serpentine" mode, in which the scan speed is increased to a value sufficient to avoid saturation of the target Cepheid in the broadband filter F606W, typically to about $1^{\prime \prime}-4^{\prime \prime} \mathrm{s}^{-1}$ (up to an order of magnitude faster than the straight scan). With such a high scan speed, the length of the scan in the standard $350 \mathrm{~s}$ exposure time exceeds the size of the detector. Thus, in order to fit the length of the scan, it is necessary to "fold" the scan itself: the telescope describes a series of parallel forward and backward scans, offset by a user-selectable amount in the $X$ direction. We use a separation of $4^{\prime \prime}$, about 100 pixels. These scans are more complex to analyze and are more affected by potential crosscontamination (overlap between scans pertaining to different stars) because of the higher density of scans, but they offer the potential for direct comparison of the Cepheid and many of the reference stars within the same filter, and they can improve the measurement precision since more pixels are covered. Discussion of the analysis of serpentine scans can be found in Section 2.6.1.

As described in Paper I, in addition to the scanned observations we also obtain short, pointed observations of the field in order to determine multiband photometry of the reference stars in several medium-band filters, including WFC3's analogs of the Strömgren filters. The photometry thus obtained, combined with infrared $J H K$ photometry from the Two Micron All Sky Survey (2MASS; Skrutskie et al. 2006), space-based photometry at 3.6 and $4.5 \mu \mathrm{m}$ from the Wide-field Infrared Survey Explorer (WISE; Wright et al. 2010), and ground-based medium-resolution spectra, is used to obtain spectrophotometric distance estimates of as many of the reference stars as possible, which is a critical step in converting the relative parallax measurement for the Cepheid target into an absolute measurement. Details on the spectrophotometric data and distance estimates are in Section 3; the estimate of absolute parallax is discussed in Section 4.

\subsection{The Case of SS CMa}

Here we present the results of the analysis for the first of these 18 targets, the fundamental-mode Cepheid SS Canis Majoris (SS CMa), with a period of 12.35 days and a mean magnitude $\langle V\rangle=9.9$ mag. SS CMa was identified as variable by Hoffmeister (1929), and a period was determined by Oosterhoff (1935). We have chosen to complete the analysis of SS CMa because it is one of the first few Cepheids for which five epochs of observations have been completed, and because 


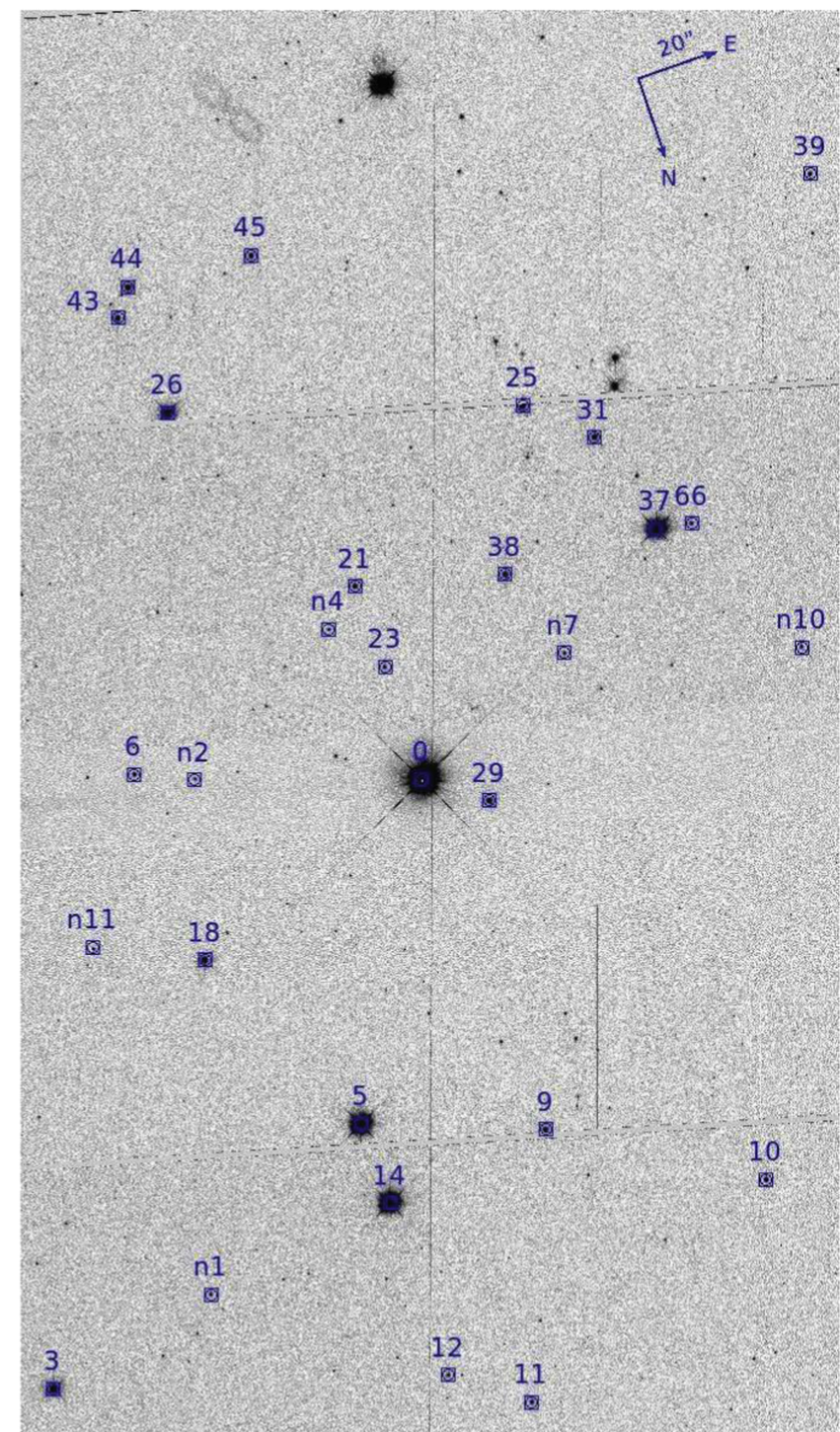

Figure 3. Mosaic of direct HST image of the field around SS CMa in filter F567M, obtained with WFC3/UVIS in $2 \times 2$ binned mode. The Cepheid at the center of the field is saturated. This image results from a mosaic of two exposures vertically displaced by $\sim 20^{\prime \prime}$ in order to cover the full field appearing in the scanned images. The two diagonal gaps result from the separation between the WFC3/UVIS detectors. Reference stars used in the analysis are marked with the identification reported in Tables 2, 3 and 4. Star 29 is the putative companion of SS CMa according to Evans \& Udalski (1994).

we believe that it is representative of the possible accuracy of the parallax measurements for the rest of the targets of our program.

Figure 3 shows a mosaic of the region of sky around SS $\mathrm{CMa}$ in the filter F547M, as obtained from the very short observations included in our program. The area represents approximately two WFC3/UVIS fields of view, stacked vertically with an overlap of $\sim 20^{\prime \prime}$. Reference stars and their designations are indicated. The Cepheid (Star 0) is saturated. The diagonal bands are caused by the gap between the two detectors in WFC3/UVIS; more observations in the future will help cover this gap. Figure 4 shows a normal scan (top) and a serpentine scan (bottom); serpentine scans are discussed further in Sections 2.4 and 2.6.1.

The Cepheid SS CMa has been discussed in the literature as a potential binary. Evans \& Udalski (1994) identify a nearby

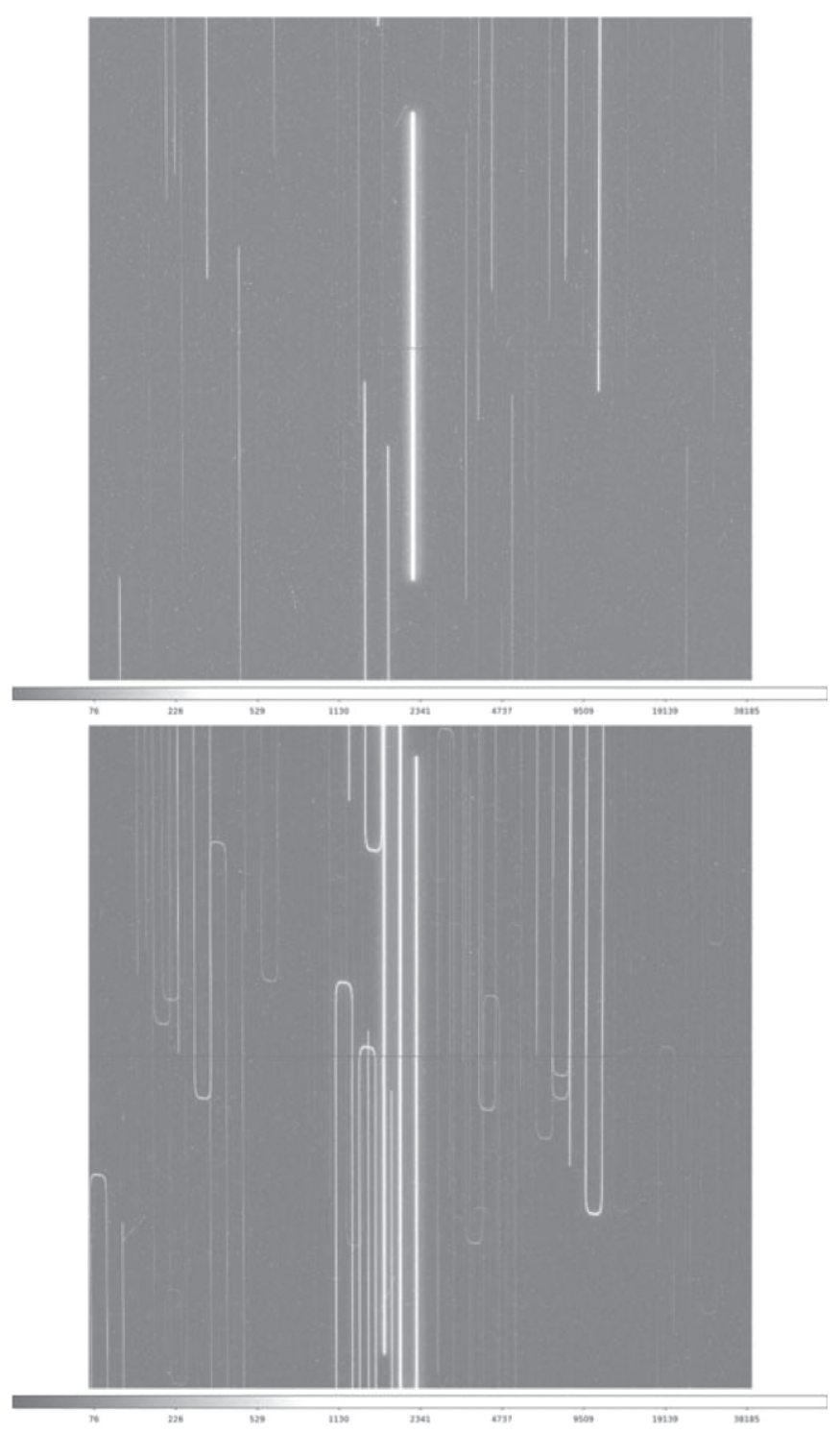

Figure 4. Top: direct scan of the SS CMa field in F621M. The central strip is the Cepheid, which is not saturated in this image. Bottom: serpentine scan obtained in F606W. For this image, the scan speed of the telescope was sufficiently high that the Cepheid does not saturate, resulting in a total scan length of over 8000 pixels. Consequently, the scan is folded twice, resulting in three separate legs for each star.

faint blue star (Star 29 in Figure 3; 13" from the Cepheid, $V \approx$ $15.51-15.58 \mathrm{mag}$ ), which they argue is likely to be a physical companion, on the basis of its estimated distance modulus and of probabilistic arguments. If Star 29 were physically bound to SS CMa, it would imply a separation much larger than the typical upper limit for physical companions ( $4000 \mathrm{AU}$; Evans et al. 2016). We will show in Section 4 that astrometric and spectrophotometric evidence suggests that Star 29 is significantly closer than the Cepheid and therefore not a physical companion. Szabados (1996) reports an apparent difference of $\sim 15 \mathrm{~km} \mathrm{~s}^{-1}$ between the radial velocities (RVs) measured by Joy (1937) and Coulson \& Caldwell (1985). R. I. Anderson et al. (2016, in preparation) also report a long-term RV change. If interpreted as caused by binarity, such changes would indicate an unresolved stellar companion on a currently unconstrained orbit with period likely on the order of decades. 
Binarity can potentially bias the astrometric parallax determination if the orbital motion of the Cepheid itself, sampled at the times of the astrometric observations, has a sufficient component to contribute to the parallax signature. This is most likely for orbits with period $\sim 1 \mathrm{yr}$; very long period binaries will produce primarily a proper-motion bias, and short period binaries have a small astrometric signature that will typically average out in the measurements.

For the case of SS CMa, we have obtained new RV measurements, discussed in detail in Section 5, which demonstrate that the contribution of binary motion compatible with the observations is most likely below a few microarseconds, thus significantly smaller than the uncertainty in our parallax measurement.

\subsection{HST Spatial Scans for Astrometry}

In Paper I we demonstrated that for sufficiently bright sources, scanning-mode HST observations, in which the telescope is slewed during the exposure and each star leaves a trail of light nearly along a pixel axis, can achieve positional measurements with a precision up to 0.5-1 millipixels (1 millipixel, or mpix, is about $40 \mu \mathrm{as}$ ) in the direction perpendicular to the scan, about a factor of 10 better than optimal measurements from pointed images. However, we also discovered that at the millipixel level, several systematic effects come into play and need to be properly calibrated in order to fully realize a corresponding accuracy of $\lesssim 40 \mu$ as in the parallax measurement.

Perhaps the most problematic systematic effect is in the insufficiently characterized, and variable, geometric distortion solution for the WFC3/UVIS camera. The mapping between pixels and the sky is well established and calibrated for direct images, with a residual uncertainty currently estimated below 0.006 pixels (rms) over the field (Bellini et al. 2011) - fully adequate for the astrometric interpretation of direct, pointed images. However, this uncertainty is an order of magnitude higher than the precision achievable in scanning mode and the requirements of our program. Calibration observations of the field of M35, obtained $1 \mathrm{yr}$ apart in 2012 and 2013, show that much of the residual geometric distortion at the sub-0.01 pixel level is static and smooth, in that the residuals vary slowly over the field of view (on scales of $\sim 100$ pixels) and are highly correlated from year to year.

Figure 5 shows that the pattern of residual geometric distortion is repeatable over short (orbital) timescales. Each line shows the variation in the measured $X$ position along the scan for the same bright star, located at the same detector position, in several consecutive scans over a two-orbit period, after subtracting the jitter pattern for that observation. To reduce pixel-to-pixel noise, the lines have been smoothed with a 20pixel length. Without residuals in the geometric distortion or other disturbance factors, these lines should all be consistent with zero (i.e., a constant $X$ position along the scan). Instead, there is a definite pattern of deviations, and this pattern is consistent from scan to scan. The horizontal dashed lines indicate the nominal precision of $10 \mathrm{mpix}$ for pointed observations, which is also the expected accuracy of the standard geometric distortion solution. This distortion pattern repeats closely even a year later: Figure 6 shows the median pattern for the same star in the same sets of observations taken $1 \mathrm{yr}$ apart. (Consistent with our treatment of all scans, an overall tilt of the lines has been solved for and subtracted.)

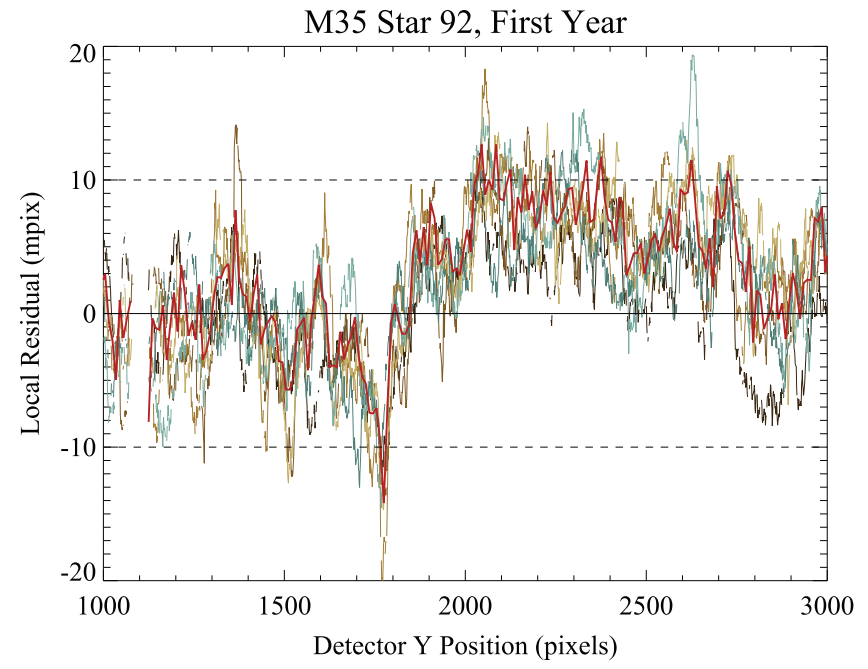

Figure 5. Repeatability of the differential geometric distortion over a two-orbit time period. The thin colored lines show the offset in the detector $X$ direction for a single bright star in M35 over repeated scans, smoothed over 20 pixels, and expressed in millipixels. The horizontal dashed lines indicate the nominal precision of the standard geometric distortion, 0.01 pixels. The thicker red line is the mean differential distortion and has an rms amplitude of 6.0 mpix; the rms difference between individual lines and the mean is 4.0 mpix. The typical statistical measurement uncertainty for this star is $\sim 2$ mpix per smoothed pixel per scan, and under 1 mpix per cell.

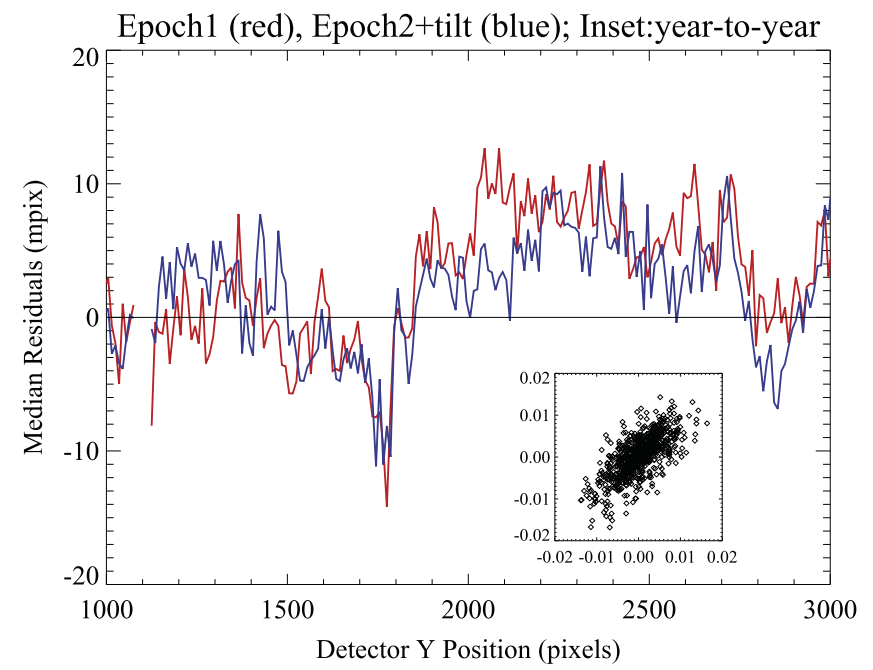

Figure 6. Comparison between the differential geometric distortion measured for the same star in the same detector location $1 \mathrm{yr}$ apart. The red line is the same as in Figure 5; the blue line is the result of the same measurement $1 \mathrm{yr}$ later, with a global tilt removed. The measurements track one another very closely, demonstrating that the local differential geometric distortion remains constant over a $1 \mathrm{yr}$ period. The rms difference between the lines is 3.9 mpix, corresponding to a repeatability of about 2.8 mpix. The inset shows the differential distortion measured in each $100 \times 100$ pixel cell in Year 1 (abscissa) vs. Year 2 (ordinate), expressed in pixels. The year-over-year correlation coefficient is 0.70 .

Again, the patterns are very similar, strongly suggesting that the residual geometric distortion is stable over time. The inset in Figure 6 shows the measured differential geometric distortion in each cell in Year 1 (abscissa) versus Year 2 (ordinate); the two quantities are highly correlated $(r \approx 0.70)$, showing that a significant part of the distortion remains the same from year to year over the whole field of view.

However, the differences between scans in the same year, or between years, are larger than nominal statistical errors, which 
are below 1 mpix per cell. Time-dependent geometric distortion, identified and discussed in Paper I for both calibration and SY Aur observations, contributes to these differences. We thus attempt to characterize and correct for both a static and a time-dependent component of the geometric distortion correction, in different ways.

\subsubsection{Static Correction to the Geometric Distortion Solution}

Even neglecting the time dependence of the geometric distortion solution, any residual static term will affect our solution. The reason is that parallax observations need to take place approximately at 6-month intervals, and orbital geometry mandates that observations 6 months apart cannot be taken at the same orientation, although they can generally be taken at orientations $180^{\circ}$ apart. Thus, for a typical target, there will be three epochs taken at one orientation, and two taken at an orientation different by $180^{\circ}$. Changing the telescope roll by $180^{\circ}$ ensures that the resolution direction, perpendicular to the scan direction and thus typically along the detector $X$ direction, remains the same on the sky, thus greatly simplifying the analysis and improving the accuracy of the results.

Target and reference stars can be placed essentially at the same detector location for each orientation; as we are interested in relative variations in the stars' position, small errors in the geometric distortion solution, which typically behave smoothly over the detector, will cancel out. However, this is not the case across orientations, when each star's location moves to a completely different place in the detector, and thus the accuracy in the geometric distortion solution comes in fully.

In order to improve the static geometric distortion solution, we have analyzed calibration observations taken of two open clusters, M48 and M67, which offer a wealth of bright stars and thus allow a dense sampling of the detector in as few as 10 dithers. Such observations have been obtained as part of the Cycle 22 WFC3 calibration program and have demonstrated that a static term, sampled on a grid of $100 \times 100$ pixel cells, can account for about half of the deviation from an accurate solution. (The differences remain larger than the statistical measurement errors, which are below 1 mpix, in part because of the time-dependent correction discussed in Section 2.3.2.) We therefore employ this solution as a correction to the default geometric distortion solution obtained by Bellini et al. (2011). The pattern of static geometric distortion thus obtained is shown in Figure 7; the top panel is for F606W, and the bottom panel is for F621M. The two patterns look remarkably similar; Figure 8 shows the strong correlation $(r \approx 0.7)$ between the geometric distortion corrections thus obtained, despite the fact that the observations targeted different star fields, in different filters, and were taken more than 1 month apart.

\subsubsection{Time-dependent Correction}

Even for observations obtained with stars at exactly the same location and in back-to-back exposures, we find that there is a smooth variation in the relative positions measured for each star, which generally can be well approximated as a low-order polynomial function of the position on the detector. This variation appears correlated with the nominal focus position of the telescope, which is provided after the fact by the Space Telescope Science Institute (STScI) Telescopes group as a result of a temperature-based model of the
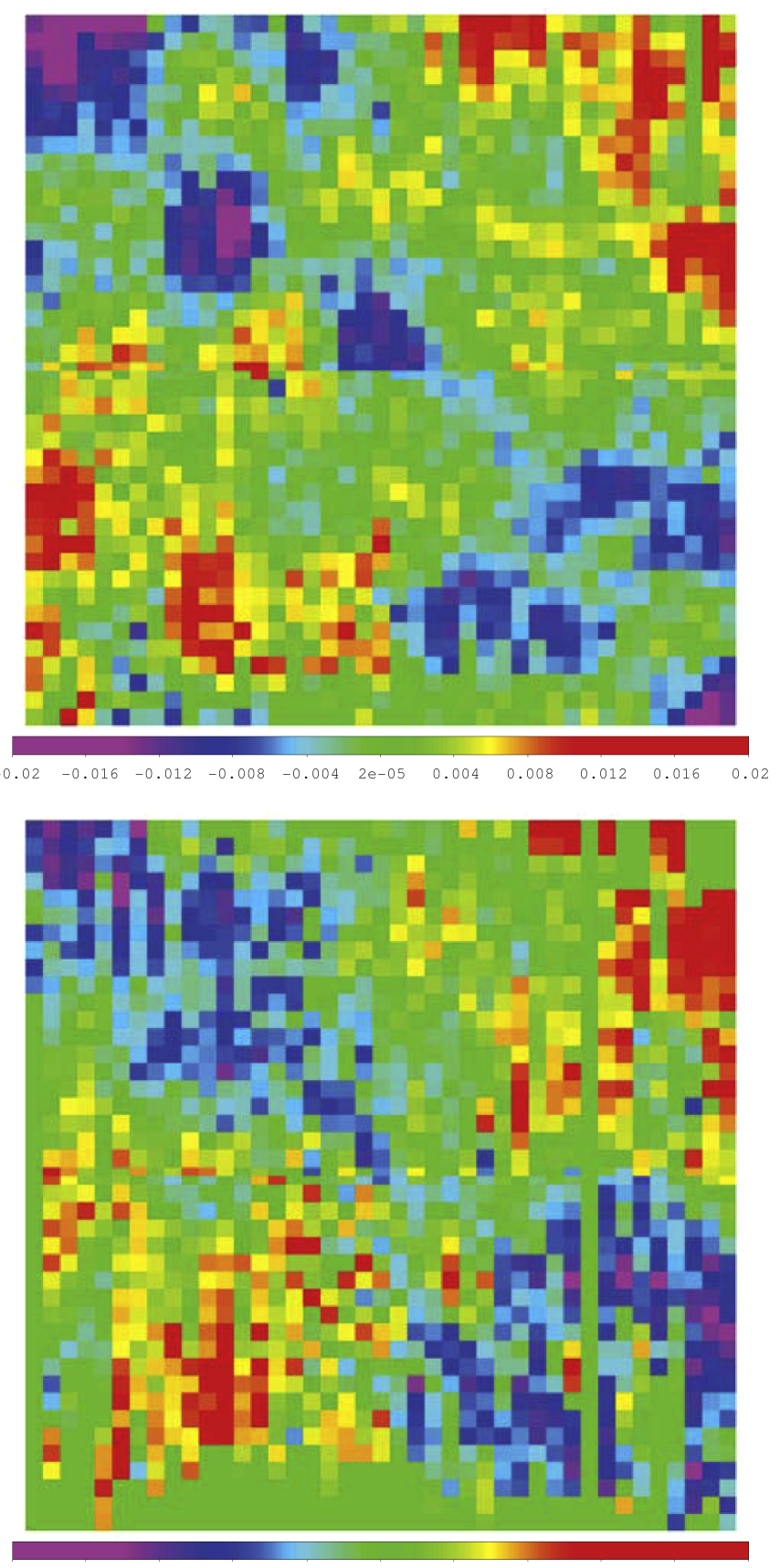

$\begin{array}{lllllllllll}0.02 & -0.016 & -0.012 & -0.008 & -0.004 & 2 \mathrm{e}-05 & 0.004 & 0.008 & 0.012 & 0.016 & 0.02\end{array}$

Figure 7. Top: pseudo-color representation of the static correction to the default geometric solution in the $X$ direction for $\mathrm{F} 606 \mathrm{~W}$, averaged over $100 \times 100$ pixel cells. The correction was obtained from scanned observations of a field in the open cluster M67. A total of $20 X$ dithers were obtained in order to cover the detector as well as possible. The color bar ranges from -0.02 to 0.02 pixels. Over most of the field of view, the correction is less than 0.01 pixels (blue to orange), but there are small regions with large corrections (negative: purple, positive: red). Bottom: same as the top panel, but for F621M, from 15 dithers in the open cluster M48 to better match the sensitivity of the narrower filter. The overall pattern is similar to F606W (see also Figure 8). About $5 \%$ of the cells have no measurement.

telescope's optical train (http://www.stsci.edu/hst/ observatory/focus/FocusModel). There is also a suggestion that the polynomial coefficients are correlated, and in fact a principal component analysis (PCA) of the correction polynomial shows that two or three parameters suffice to account for over $95 \%$ of the correction. 


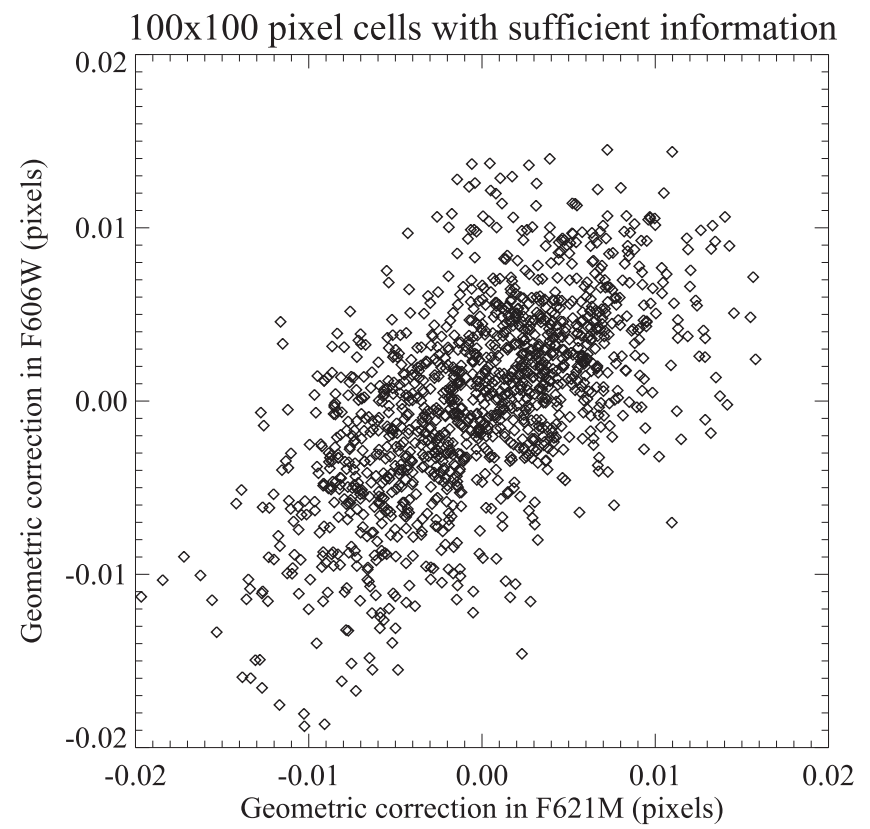

Figure 8. Comparison between the geometric distortion residuals in F621M and F606W, respectively, for each $100 \times 100$ pixel cell in the detector. There is a clear correlation between the residuals, indicating that the bulk of the correction is in common between filters. However, about $30 \%$ of the residual correction, as determined from these measurements, differs between the filters, suggesting that a better correction is achieved with a filter-specific correction.

Although the correlations of the polynomial correction with focus and internally across coefficients are highly indicative, we do not yet have sufficient information to characterize the required correction directly from estimated focus. We therefore resort to a self-calibration approach, in which the polynomial distortion is part of the model and is chosen so as to minimize the source-by-source residuals of the full set of observations across epochs.

\subsection{Designing the Observations}

Based on our experience observing the field of SY Aur (Paper I), we developed simulation tools to optimize the observations of other Cepheids, and we applied these to the field of SS CMa. As in SY Aur, we selected positions, scan speeds, scan lengths, and filters to allow the highest-quality parallax measurements from the field (see Paper I for these details). The field of SS CMa provided more than twice as many reference stars of intermediate brightness, used to register the deep and shallow scans, as the field of SY Aur.

In addition to the straight scans we discussed in Paper Itwo each in broadband and narrowband filters at each epochwe have also obtained and processed serpentine scans, in which the telescope moves through the field in a boustrophedonic pattern (down to up, shift right, up to down, shift right, etc.), as shown in the bottom panel of Figure 4 . With this pattern scanned at a rate of $1{ }^{\prime \prime} 5 \mathrm{~s}^{-1}$, the target Cepheid does not saturate even in the broadband filter. However, at this rate the telescope will traverse about $525^{\prime \prime}$ over a $350 \mathrm{~s}$ exposure, almost four times the WFC3 field of view. Taking exposures shorter than $350 \mathrm{~s}$ is not desirable because of how memory is managed in WFC3; therefore, in order to keep the target and most of the reference stars on chip for the largest fraction of the time, and thus to collect as many photons as possible, the scan pattern is folded into multiple near-straight scan lines, all approximately along the $Y$ direction, with small "crossbars" between them. In the case of SS CMa, the F606W serpentine scans had three legs, and the overall length was such that no crossbars are visible for the Cepheid; the turnaround occurred while the Cepheid was off-chip. Owing to planning priorities, no serpentine scan was obtained in the fifth and final epoch.

The advantage of the serpentine scanning pattern is that the Cepheid and most of the reference stars are observable in the same exposure; thus, it is possible to solve directly for relative and absolute parallaxes without the potential for inconsistencies in the geometric distortion solution across filters. The disadvantages are that (1) the telescope scans faster than in the narrowband frame with equivalent count rate, therefore providing less local contrast against the sky background for faint reference stars (this is partially compensated by the larger number of points per star); (2) the exposures are more crowded, with a greater chance that otherwise fine reference stars will be marred by an overlapping trace from a different star; and (3) the motion of the telescope is more complex, and it is more difficult to identify fixed points such as the start and end of each scan. For example, for SS CMa the scan length in the middle leg exceeds the size of the WFC3 instantaneous field of view, so that neither its start nor its end is visible. Thus, it is more challenging to determine the position of the reference points along each leg, other than the overall start and end. These difficulties notwithstanding, we have been able to process serpentine scans with methods similar to our other scans, and the results are incorporated in our astrometric solution (see Section 2.6.1).

\subsection{Analysis of Scan Data}

The analysis of the scan data for SS CMa largely follows the pattern we described in Paper I for SY Aur. The key steps are (1) identifying the pixels associated with each trail (star); (2) defining a minirow-by-minirow detector $X$ position at each location along the trail by a one-dimensional fit of the observed signal along each minirow with a spatially variable line-spread function (LSF); (3) converting the position into rectified coordinates using the distortion map; (4) removing the effects of jitter and variable rotation; (5) determining the relative rectified $X$ position for each star in each image; (6) combining the measurements from multiple scans within each epoch, including both deep and shallow frames; and, finally, (7) estimating the parallax of each target on the basis of the combined astrometric and spectrophotometric information. Key differences in the processing for SS CMa are (1) the availability of an improved geometric distortion solution via the static correction discussed in Section 2.3.1; (2) the use of serpentine scans, which are combined with deep and shallow frames for each epoch; and (3) the introduction of an empirical correction for the $X$-direction CTE loss (X-CTE). Thanks to the number and quality of reference stars, the nominal error of the parallax for SS CMa is significantly smaller than for SY Aur. We will now discuss in detail each step, highlighting the changes with respect to Paper I.

Our astrometric measurements are based on the spatial scan exposures listed in Table 5; Figure 4 shows typical examples of straight and serpentine scans.

The nearly vertical "trails" are the images that each star leaves as the telescope scans over the field. The length of the straight scans is $\approx 144^{\prime \prime}, 88 \%$ of the length of the field of view of WFC3/UVIS; thus, the part of the sky covered during the 
scan is almost twice the normal field of view of the camera. Stars near the center of the region spanned in the detector $Y$ direction will have trails that start and end within the frame, while stars farther from the center along $Y$ have trails that enter or leave the frame during the scan. For serpentine scans, the telescope motion is more complex; the vertical portions are scanned at 1 !" $4 \mathrm{~s}^{-1}$, about 35 pixels $\mathrm{s}^{-1}$, a speed chosen to avoid saturation of SS CMa in filter F606W. However, at that scan speed, and given the desired exposure time of $350 \mathrm{~s}$, the total length of the scan is $\approx 525^{\prime \prime}$, over three times the field of view of the detector. Therefore, the scan is folded, consisting of three vertical legs separated by $4^{\prime \prime}$ in the detector $X$ direction. The turnarounds for the Cepheid occur just outside the detector field of view; other stars, at lower (higher) $Y$ position, can have their first (second) turnaround within the field of view.

Note that all celestial sources in the field are extended because of the motion of the telescope. Cosmic rays are the only compact sources in the frame and are readily identified by their lack of spatial extent, allowing us to identify and disregard impacted pixels.

We used a master catalog of stars to first simulate and then match the observed trails to these stars. The fidelity of the simulations is a few pixels, and in many cases minor adjustments are needed to ensure that the full 15-pixel window around each trail pixel is fitted. We also use the simulations to identify the regions within each star's trail that are affected by nearby star trails, and we disregard the impacted pixels if the simulation indicates a bias of greater than $1.5 \mathrm{mpix}$ for a given row.

The case of serpentine scans is more complex. For serpentine scans, each star's trail is marked by multiple reference points: the overall start and end, which are the start of the first leg and the end of the last leg, and whose location is determined by the shutter opening and closing; and the turnarounds between legs, whose location is determined by the motion of the telescope. For any given star, only a subset of these points is visible in the image. Furthermore, we have found that the relative location of these reference points cannot be predicted with sufficient precision from image to image, and therefore it has to be determined empirically from the data themselves. Locating individual trails in a serpentine image is thus a two-step process: first, locate the start, end, and turnaround points for a subset of well-exposed stars at various locations in the field of view; second, determine the geometry of the scan (with an accuracy of $\sim 3$ pixels) and apply this geometry to predict the serpentine trails of all the stars visible in the scan using our star catalog. The latter include stars too faint to be profitably measured, but that are still bright enough to "spoil" the scans of brighter stars, as described above. The trail map must then be inspected and reference points tweaked to improve the match with the data; at the end of this labor-intensive process, we were generally able to identify and locate the trails of individual stars to within $\sim 1$ pixel.

For the purpose of subsequent analysis, trails in serpentine scans are split into individual passes, each including the trails of all relevant stars in that pass. This allows us to associate together photons collected at the same time and consistently solve for time-dependent effects, such as the variable field rotation discussed below. It also allows for a more careful identification of spoilers, since different legs can have different spoiler impact. In the case of SS CMa, each three-leg serpentine scan results in three separate position measurements for all the stars, thereby associating together measurements taken at the same time. Note that the serpentine scan was not obtained during the fifth epoch; thus, Epochs 1 through 4 each have seven sets of measured positions (five for the Cepheid), while Epoch 5 has four sets of positions (two for the Cepheid).

As in Paper I, we independently fit each 15-pixel minirow along the trail to determine the $X$ position of the star at that value of $Y$. The fit uses an empirical LSF appropriate to the filter and detector position, obtained by integrating the empirical point-spread functions (PSFs) from Bellini et al. (2011) and, like the latter, oversampled by a factor of 4 . Data quality flags from the detector characterization, as well as flags from source-contaminated pixels, are used to avoid fitting bad pixels. The end result is an array of detector $X$ positions and uncertainties as a function of the $Y$-axis position (equivalent to time) along the scan.

Also following the same procedure as in Paper I, we start with the geometric distortion solution for F606W from Bellini et al. (2011), which uses a definition of the PSF position that is consistent with the empirical determination of the PSF itself. This geometric distortion map is used to transform the detector $X$ and $Y$ positions to sky coordinates. We obtain a similar solution for F621M from calibration observations of $\omega$ Cen. In addition, in this paper we also correct for the residual geometric distortion obtained from calibration observations of M67 and M48 (Section 2.3.1). The original solution from Bellini et al. (2011) is expected to have an accuracy of $\sim 0.01$ pixels on scales of $\sim 40$ pixels; this accuracy is sufficient to reach position precision of $1 \mathrm{mpix}$ (40 $\mu$ as) for full-length scans, which would be a significant contribution to our overall error budget. By applying our new correction, we expect that the local residuals will be reduced to $\sim 3$ mpix on a scale of 100 pixels, with a projected contribution to the final error budget of $\sim 20 \mu$ as. Again as in Paper I, we use the time-dependent velocity aberration values provided by the STScI pipeline, interpolated to account for its variation during the observation, to correct for the corresponding plate-scale changes along the scans. Later, we account for perturbations in the geometric distortion of the field caused by the day-night thermal cycle of HST when registering different scans.

As for the analysis of SY Aur, we define the onedimensional position measurements for each scan line relative to the mean line of the sample. This mean line or reference line is determined by aligning all scan lines in time and taking their weighted average. The reference line thus contains the jitter history in the direction perpendicular to the scan, which is removed from all lines in their difference with the reference. Figure 9 shows the comparison of two bright star trails aligned in scan time and the residuals after subtraction of one from the other. We use the requirement that scan lines, relative to the reference, are parallel on the sky to measure the time dependence of the scan roll angle. The variable rotation history of the scans can be measured well for the two deep scans obtained at each epoch in F606W; Figure 10 shows that the rotation angle is very similar in both scans obtained at the same epoch. The variable rotation cannot be measured as accurately in the shallow scans, because of the lower signalto-noise ratio for all stars except for the Cepheid, which by itself cannot constrain the rotation angle. As the variable rotation term appears to be constant within each epoch, we determine the correction by averaging the measured rotation 


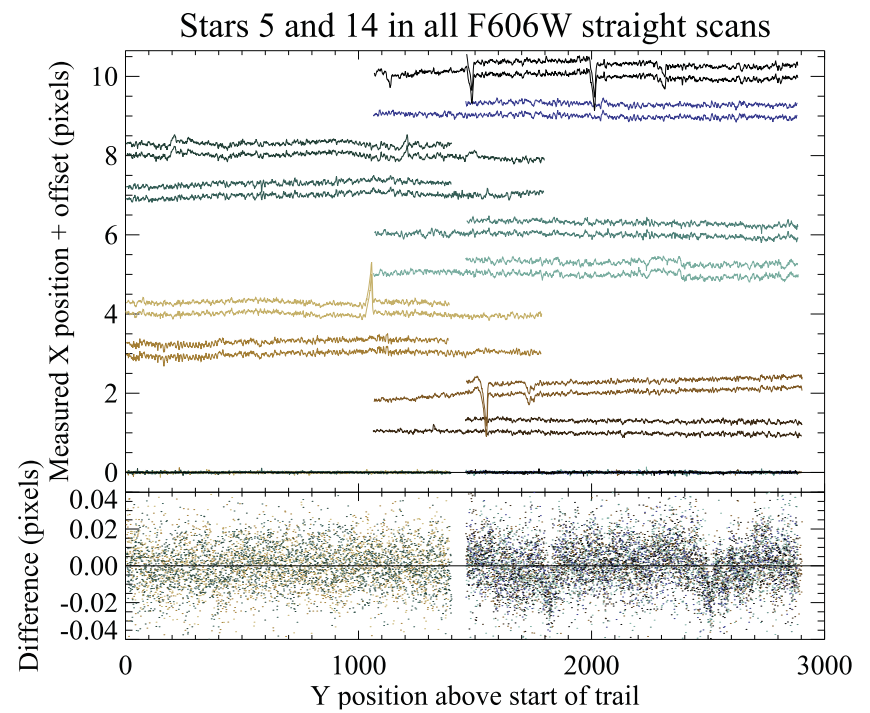

Figure 9. Variations in the measured $X$ position for two bright stars (Stars 5 and 14) in the field of SS CMa in each of the 10 full-length straight F606W scanned exposures. The top panel shows the individual X measurements, with a different color for each exposure. Scans have been offset along the abscissa to match photons received at the same time, and along the ordinate by an arbitrary constant. The very high correlation between the irregularities in the two scans shows that most of the apparent "noise" in the measured position is actually telescope jitter. The differences between the two stars in each exposure are overplotted at $X \approx 0$, and again in the bottom panel with a scale expanded by a factor of 50. The pixel-to-pixel variation in the difference is consistent with the expected uncertainty in the fit for each minirow; the nominal error in the mean separation in each exposure is 0.4 mpix, or $16 \mu$ as.

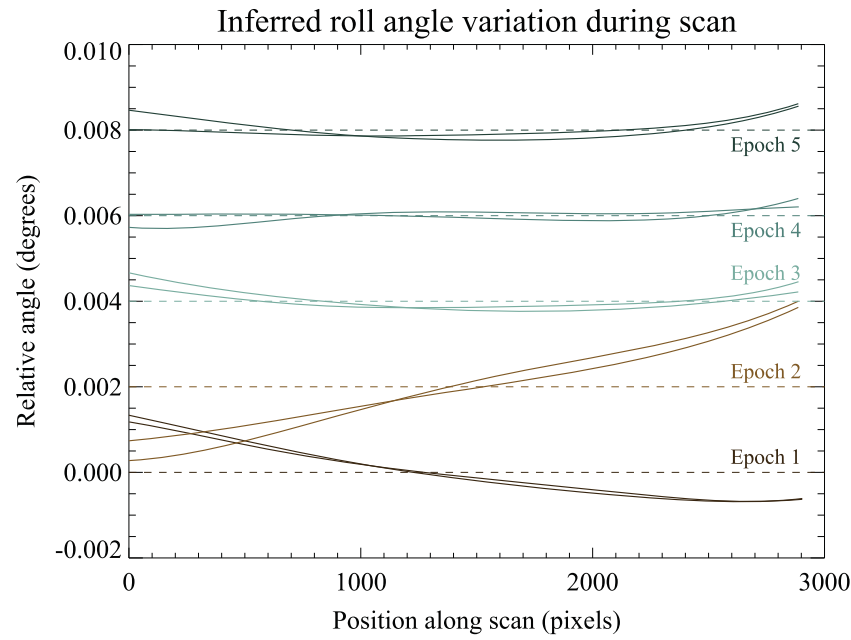

Figure 10. Differential field rotation during the F606W scans in each of the five epochs of the SS CMa observations, represented as a fifth-degree polynomial as a function of position along the scan. A correction for the differential field rotation is applied to the measured $X$ positions along each scan. Note the similarity of the pattern for the two observations obtained at each epoch, as well as the marked decrease in the change starting in Epoch 3, after an improved FGS distortion solution was adopted in 2013 July.

in the two deep scans at each epoch, and we apply the resulting correction to all deep and shallow scans for that epoch. Figure 10 also shows that the variable rotation is markedly smaller in Epochs 3 through 5, most likely because of the improved FGS geometric solution adopted on 2013 July 22, between our Epochs 2 and 3 (E. Nelan \& M. Lallo 2016, private communication).

\subsection{Using Multiple Observations at the Same Epoch}

The fundamental measurements at each epoch of observation consist of the relative $X$ position of all stars, obtained from the combination of all coeval scans, deep, shallow, and (if available) serpentine. In order to combine these scans, they must be astrometrically registered, which requires correcting for any differential geometric distortion. As in Paper I, we include in the distortion model a low-order polynomial correction with free coefficients for each observation after the first. We adopt a polynomial correction to the $X$ coordinate when aligning two frames that depends on the pixel position of each star trail in the detector; the assumption is that any variation in the transformation from true to measured position is tied to the telescope and detector and therefore is best described in measured rather than true coordinates. Note that a generic first-degree polynomial includes by definition an $X$ scale term (the first-order correction in $X$ ), as well as a detector rotation, which is slightly different from but closely related to the field rotation previously considered. As for M35 and SY Aur, we find that a second-degree polynomial as a function of $X$ and $Y$ coordinates is adequate to describe the $X$-coordinate transformation between two scans. (Only terms of total degree up to the polynomial degree are included, so our second-degree polynomial contains terms in $X \times Y$, but not in $X^{2} \times Y$ or $X \times Y^{2}$.) A second-degree polynomial in $X$ and $Y$ has five coefficients (plus a constant term), two of which describe an offset and a rotation, respectively.

For SY Aur we found that a dearth of intermediatebrightness stars, combined with the need to allow for secondorder polynomial corrections between the frames, led to significant uncertainties in the transformation of the Cepheid and of the reference stars to a common frame, resulting in a dominant contribution to the final uncertainty.

In order to ameliorate this problem, we modified our strategy in two ways. First, the number of available intermediatebrightness reference stars was a primary consideration in the selection of our targets. Second, we have obtained and processed serpentine scans in $\mathrm{F} 606 \mathrm{~W}$; these scans provide additional constraints between the Cepheid, which is not saturated, and a larger number of intermediate-brightness reference stars. (Similar data were collected in some of the SY Aur epochs, but we were unable to process them properly for Paper I.) Position measurements for intermediate-brightness and faint stars in serpentine scans are not quite as accurate as those in the straight F606W scans, for a number of reasons: the same total counts are spread over a larger area, thus resulting in additional background noise; the higher density of trails results in more spoilers; and the more complex telescope motion results in additional parameters to be fitted. Nonetheless, we find that serpentine scans, while more complex to analyze, add significantly to the precision and reliability of the final measurement, and we expect that the analysis of future targets will use the serpentine scans as well.

To account for a shift in detector $X$ position due to imperfect $\mathrm{X}$-CTE, we have obtained calibration observations for WFC3/ UVIS in spatial scan mode, in which a bright star has been moved across the amplifier boundary at $X=2048$ in consecutive exposures. To the extent that the X-CTE effect is linear in the distance to the relevant amplifier, the mean relative position of stars in the field does not change as the field of view is dithered in the $X$ direction-if stars are moved to the right ( $+X$ direction), those to the left of the boundary $(X<2048)$ 
will experience an increase in their apparent displacement to the right, and those to the right of the boundary $(X>2048)$ will experience a decrease of their apparent displacement to the left, for a null net effect. (Individual stars will move slightly with respect to one another, as fainter stars will be affected more than brighter stars.) However, if a star is moved from left to right of the boundary, its apparent displacement due to X-CTE will reverse sign, and thus it will experience a large net motion. An analysis of calibration observations obtained in Fall 2014 shows that the net effect is 8 mpix for a star near saturation, thus implying that a bright star near the amplifier boundary was shifted by 4 mpix at that time owing to X-CTE.

Experience with WFPC2, ACS, and (more limited) WFC3/ UVIS strongly suggests that CTE effects grow linearly with time in orbit and with distance to the relevant amplifier. For WFC3/UVIS, we assume that the CTE loss was zero at launch. If this is correct, and if the effect is furthermore antisymmetric with respect to the amplifier boundary, the impact of X-CTE on parallax determinations vanishes as long as the observations are obtained with the same center and rotated field of view. The reason is that a growing X-CTE will result in an apparent motion for each star that is indistinguishable from a proper motion; thus, each star will have a spurious term in its estimated proper motion, but the parallax estimate is unaffected. Even if the field of view is shifted, the impact on each star is minimal, as discussed above-as long as the star does not switch amplifiers.

However, second-epoch observations placed the Cepheid at the same $X$ detector location $(X \approx 2000$, left of the amplifier gap) as the odd epochs, thus shifting the field center by about 100 pixels between the two orientations. The aim was to minimize the impact of uncertainties in the geometric distortion for the Cepheid by placing it in approximately the same detector location. The magnitude of the X-CTE effect was not fully understood at that time. On the basis of current information, minimizing the impact of X-CTE effects is deemed more important, and starting from Epoch 3, observations were obtained with the same field center for all epochs.

In order to correct for the residual X-CTE effect-which generally only affects the Cepheid target, as other stars within 50 pixels of the amplifier boundary are swamped by the light of the target-we simply correct the position measured for the Cepheid in Epoch 2 by twice the estimated offset at that time, about 2.9 mpix. This correction mimics the effect of placing the Cepheid in the symmetrical position $(X \approx 2100)$ and thus nullifies the effect of X-CTE on the measured parallax. As discussed above, relative proper motions will be affected by $\mathrm{X}-\mathrm{CTE}$ and thus can only be determined accurately if a good overall calibration for X-CTE is obtained.

\subsubsection{Serpentine Scans}

Although in many ways the serpentine scans are treated similarly to the regular straight scans, some special considerations apply. For each leg, we exclude from the fit a region of about 300 pixels before and after each turning point, in which the motion of the telescope deviates significantly from a straight line. In principle, we could include this deviation in the overall fit; however, the local slope can be large enough that our underlying approximation that the scan direction is perpendicular to the resolution direction no longer fully applies. For such regions, the variable-rotation solution (see Section 2.4) would also fail its underlying assumptions.
Therefore, we simply ensure that we only include the portion of each leg where the mean displacement of the motion from a straight line is less than 0.1 pixels.

In addition, serpentine scans do not provide a good way to determine the start or end point of each leg accurately during the initial fit. The half-rise method does not work, as each leg is truncated by the cutoff in the horizontal offset, rather than by the rise or fall due to the shutter. Only the very first and last leg could have a half-rise measurement, and only if the relevant start/end point occurs on chip. For this reason, we put special care in estimating the start and end point from the overall shape of the serpentine scan, and we use these start and end points for the initial guess at the vertical positioning of each scan. In keeping with our procedures for straight scans, a refinement step occurs in which a least-squares fitting of the jitter pattern along the scan is used to improve the relative positioning of each scan. This step works to a similar accuracy as for the straight scans, after taking into account the relative signal levels and scan lengths.

\subsection{Combining Multi-epoch Data: Toward a Parallax Measurement}

For each epoch of observation, our goal is to obtain a measurement of the relative positions of all stars, the Cepheid as well as all the reference stars, along the resolution direction (the distortion-corrected detector $X$ axis projected onto the sky). This measurement must be as accurate and free of systematics as possible.

In order to obtain a measurement of the relative parallax and proper motion (in the resolution direction) of the stars on the field, data from all available epochs must be combined. In addition, information on the distance of the reference stars is required in order to obtain an absolute parallax for the target. In principle, the process requires only a linear combination of the positions as measured at each epoch to determine relative parallaxes and proper motions; if the mean parallax of the reference stars can be estimated, determining the parallax of the target is then straightforward.

In practice, solving for the parallax of the target is much more complex. Small rotations (at the level of a few hundredths of a degree) between epochs, as well as small changes in plate scale and low-order geometric distortion that are known to occur, can substantially affect the projected positions of each star in each epoch and thus impact significantly its estimates of parallax and proper motion. Occasionally, reference stars can have anomalous data, either because of measurement problems (e.g., undetected faint stars close enough to affect the fit) or for astrophysical reasons (e.g., binary companions or other sources of photocenter motion).

The approach we have adopted solves simultaneously for the astrometric parameters of all the stars in the field and for the geometric registration of all the epochs, using the spectrophotometric parallax estimates as priors for their astrometric parallax. With this approach, the spectrophotometric parallax estimates help constrain the registration between epochs, including the relative low-order geometric distortions. It is thus critical that the parallax estimates be as accurate and robust as possible.

In the next section, we present the combination of spectroscopic and photometric data and the analysis that leads to spectrophotometric distance estimates for as many of the reference stars as possible. 
In Section 4 we return to the determination of the Cepheid parallax using the spectrophotometric distance estimates obtained in Section 3.

\section{SPECTROPHOTOMETRIC DATA AND DISTANCE ESTIMATES}

\subsection{Photometry and Spectroscopy of Reference Stars}

Narrow-angle astrometry, such as what we can obtain with $H S T$, is fundamentally differential in nature, and therefore it can only constrain the difference between the parallax of stars within the field of interest. In order to convert this relative parallax estimate into an absolute measurement, the parallax of other stars in the field must be estimated, and careful consideration must be given to possible systematics and random uncertainties in these estimates. Following the approach used in previous studies (e.g., Harrison et al. 1999; Benedict et al. 2007), we estimate the individual distances of several reference stars with respect to which the relative parallax of our target is measured in order to convert its value to an absolute parallax. High-quality distance estimates, based on both spectroscopy and multiband photometry, and careful consideration of the uncertainties involved are paramount in obtaining a reliable, accurate conversion to absolute parallax. For this purpose, we have obtained medium-resolution, classification-quality spectroscopy and multiband photometry ranging from $\mathrm{UV}$ to mid-infrared, including a number of medium-band Strömgren-like filters, for all the reference stars in the field of SS CMa. A combination of stellar model fitting and spectrophotometric classification, together with an understanding of the distribution of stars along the line of sight, has been used to estimate the distance to each reference star and its likely uncertainty. We also used prior estimates of the reddening along the line of sight, based on measurements of stars in 2MASS and Pan-STARRS, in order to constrain the range of possible reddening; however, the final reddeningdistance law was also fitted for in our analysis.

With this information, the relative parallaxes of the reference stars and of the Cepheid can be placed into an absolute frame. In addition, estimates of the parallaxes of other stars in the field can confirm the quality of the astrometric measurements, identify outliers, and help constrain some of the low-order geometric distortion variations discussed earlier.

\subsubsection{Photometry for Reference Stars}

For the SS CMa field (and for other Cepheid fields in progress), we obtained direct imaging with HST during the scanning observations and measured photometry of all reference stars in the UV (F275W, F336W), Strömgren (F410M, F467M, F547M), and broadband (F850LP) systems. In order to obtain the photometry efficiently within the observing time available to our program, we used a $2 \times 2$ binned mode, in which full-field WFC3/UVIS images are binned on board before being saved to the HST computer for download. In this mode, images have a substantially smaller memory footprint, and more images can be obtained before the instrument memory is full and the images must be transferred to the HST solid-state storage. Consequently, we were able to obtain several photometric measurements within each orbit, without impacting the scanning-mode observations. We have developed and tested procedures to accurately recover and calibrate binned-mode photometry, and we obtained reliable photometry (albeit with larger uncertainties) even for partially saturated stars.

We also obtained F160W photometry with WFC3/IR, and we added $J$-, $H$-, and $K$-band photometry from $2 \mathrm{MASS}$, as well as Channel 1 and Channel 2 photometry from WISE when available, to provide a set of up to 14 bands of photometry from 0.2 to $4.5 \mu \mathrm{m}$. All of the photometry was of high signal-tonoise ratio, with the exception of $\mathrm{F} 275 \mathrm{~W}$, where only a third of the stars yielded a measurement $(\mathrm{F} 275 \mathrm{~W}<22.8 \mathrm{mag})$. Missing or excluded photometry was recorded for stars that suffered cosmic-ray hits, for stars that suffered blending in the 2MASS or WISE data (as identified from their data quality flags or from HST F850LP imaging), and for half the field not covered by F410M imaging. The resulting photometric information is reported in Tables 2 and 3.

\subsubsection{Spectroscopy of Reference Stars}

We independently determined the temperature and luminosity class of the majority of the reference stars via mediumresolution optical spectra compared to template spectra. As indicated in Table 2, spectra were obtained with the Kast double spectrograph (Miller \& Stone 1993) on the $3 \mathrm{~m}$ Shane reflector at Lick Observatory, with GMOS on Gemini South (Hook et al. 2004), and with LRIS on Keck (McCarthy et al. 1998). Standard procedures were used for the data reduction.

\subsubsection{Estimating Spectrophotometric Parallaxes}

Spectroscopic parallaxes of stars in the field were determined, as in Paper I, by matching up to 14 bands of photometry to stellar isochrones, comparing medium-resolution spectroscopy to stellar spectra for classification standards, and using the Besançon Galaxy Model (Robin \& Crézé 1986; Robin et al. 2003, and references therein) as a likelihood prior for stellar parameters. We used a version of the model with an updated thick disk that better fits Sloan Digital Sky Survey (SDSS) and 2MASS data (A. Robin 2013, private communication).

Our procedure for measuring the spectroscopic parallaxes of the astrometric reference stars in the field has been somewhat refined and improved since the procedure used for the field of SY Aurigae described in Paper I. We have now added photometry of the stars from two bands of spatial scanning, a broad (F606W) and another Strömgren (F621M) filter, one broad band from HST in the near-infrared (F160W), and two bands of medium-infrared data from the all-sky WISE mission. We add an uncertainty of $0.05 \mathrm{mag}$ in quadrature to all photometric uncertainties to account for possible differences in photometric systems between models and observations. The stellar classification of star temperature and luminosity class is now done using the MKCLASS version 1.7 automated Morgan-Keenan classifier (Gray \& Corbally 2014). Finally, we have improved our prior knowledge of the extinction along the line of sight as a function of distance using the 2MASS determinations from Marshall et al. (2005), who provided extinction versus distance estimates for the line of sight in the direction of SS CMA (private communication) with an uncertainty of $0.3 \mathrm{mag}$ at a given distance. An example of the quality of the results is shown in Figure 11 for Star 18; the observed photometry (diamonds) is matched to a reddened model (dashed line), with the residuals shown on a larger scale in the bottom panel. The inset shows the observed spectrum for 
Table 2

Properties of the Reference Stars. I. UVIS Photometry

\begin{tabular}{|c|c|c|c|c|c|c|c|c|}
\hline Star & $\begin{array}{c}\text { F275W } \\
\text { WFC3/UVIS }\end{array}$ & $\begin{array}{c}\text { F336W } \\
\text { WFC3/UVIS }\end{array}$ & $\begin{array}{c}\text { F410M } \\
\text { WFC3/UVIS }\end{array}$ & $\begin{array}{c}\text { F467M } \\
\text { WFC3/UVIS }\end{array}$ & $\begin{array}{c}\text { F547M } \\
\text { WFC3/UVIS }\end{array}$ & $\begin{array}{c}\text { F606W } \\
\text { WFC3/UVIS }\end{array}$ & $\begin{array}{c}\text { F621M } \\
\text { WFC3/UVIS }\end{array}$ & $\begin{array}{c}\text { F850LP } \\
\text { WFC3/UVIS }\end{array}$ \\
\hline 3 & $\ldots$ & $\ldots$ & $\ldots$ & 2011 & 0 & $13.850=$ & 003 & 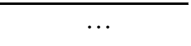 \\
\hline 5 & $13.316 \pm 0.300$ & $12.768 \pm 0.302$ & $12.977 \pm 0.301$ & $\ldots$ & $\ldots$ & & & $12.213 \pm 0.300$ \\
\hline 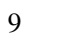 & $19.279 \pm 0.064$ & $.520 \pm 0.020$ & $.310 \pm 0.015$ & $16.685 \pm 0.012$ & $\cdots$ & $15.589 \pm 0.005$ & $15.403 \pm 0.003$ & $4.251 \pm 0.00$ \\
\hline 10 & $\ldots$ & $\ldots$ & $\ldots$ & $17.336 \pm 0.016$ & $16.638 \pm 0.006$ & $16.333 \pm 0.005$ & $16.151 \pm 0.003$ & $\cdots$ \\
\hline 11 & $\ldots$ & $\ldots$ & $\ldots$ & $17.766 \pm 0.020$ & $17.002 \pm 0.007$ & $16.662 \pm$ & $16.462=$ & $\ldots$ \\
\hline 18 & $16.308 \pm 0.014$ & $5.600 \pm($ & $15.920 \pm 0$ & $15.630 \pm 0.007$ & $15.078 \pm 0.004$ & $14.857 \pm 0.005$ & 14.724 & $13.872 \pm 0.004$ \\
\hline 21 & $19.246 \pm 0.066$ & $17.989 \pm($ & $17.361 \pm 0$ & $16.875 \pm 0.011$ & $16.328 \pm 0$ & $16.061=$ & 15.905 & $14.954 \pm 0.007$ \\
\hline 23 & $20.202 \pm 0.133$ & $18.824 \pm 0$ & $18.762 \pm 0.032$ & $18.149 \pm 0.021$ & $17.456 \pm 0.009$ & $17.081=$ & 16.902 & $15.887 \pm 0.011$ \\
\hline 25 & $18.282 \pm 0.038$ & $16.498 \pm 0.012$ & $16.250 \pm 0.009$ & & $\ldots$ & $14.725 \pm 0.004$ & 14.539 & $13.532 \pm 0.300$ \\
\hline 26 & $16.557 \pm 0.016$ & $15.474 \pm 0.007$ & $14.790 \pm 0.004$ & $14.689 \pm$ & $14.000 \pm$ & $13.716 \pm 0.003$ & $13.565 \pm 0.005$ & $12.544 \pm 0.300$ \\
\hline 29 & $18.110 \pm$ & $16.429 \pm$ & $16.524 \pm$ & 16.003 & $15.571=$ & & 004 & $14.658 \pm 0.0$ \\
\hline 43 & $\ldots$ & $\ldots$ & $\ldots$ & $17.291 \pm 0.014$ & $15.921 \pm 0.004$ & .004 & 0.006 & $\ldots$ \\
\hline 44 & $\ldots$ & $\cdots$ & $\cdots$ & $15.974 \pm 0.007$ & $15.551 \pm 0.003$ & $339 \pm 0.005$ & 0.009 & $\ldots$ \\
\hline 45 & $\ldots$ & $\ldots$ & $\ldots$ & $17.246 \pm 0.014$ & $16.137 \pm 0.005$ & $15.588 \pm 0.004$ & $15.314 \pm 0.008$ & $\cdots$ \\
\hline 66 & $20.564 \pm 0.160$ & $19.048 \pm 0.042$ & $18.896 \pm 0.034$ & $18.180 \pm 0.022$ & $17.464 \pm 0.009$ & $17.085 \pm 0.008$ & $16.868 \pm 0.009$ & $15.683 \pm 0.0$ \\
\hline $\mathrm{n} 1$ & $\cdots$ & $\ldots$ & $\ldots$ & $18.424 \pm 0.028$ & $17.785 \pm 0.011$ & 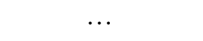 & $\cdots$ & $\ldots$ \\
\hline $\mathrm{n} 2$ & $20.976 \pm 0.419$ & $19.646 \pm 0$ & $19.513 \pm 0$ & $18.884 \pm 0.021$ & $18.073 \pm 0.009$ & $17.673 \pm$ & $17.469 \pm$ & $16.315 \pm 0.01$ \\
\hline $\mathrm{n} 4$ & $23.488 \pm 1.397$ & $20.552 \pm 0$ & $20.027 \pm 0$. & $18.979 \pm 0$ & & & .009 & $15.518 \pm 0.0$ \\
\hline n7 & $20.451 \pm 0.166$ & $19.202 \pm 0$ & $\cdots$ & & & & 014 & $15.972 \pm 0.0$ \\
\hline $\mathrm{n} 10$ & $23.207 \pm 1.152$ & $20.534 \pm 0.104$ & $19.766 \pm 0.056$ & $18.645 \pm 0.027$ & $17.626 \pm 0.010$ & $17.109 \pm 0.008$ & $16.847 \pm 0.006$ & $15.174 \pm 0.0$ \\
\hline $\mathrm{n} 11$ & $21.637 \pm 0.790$ & $20.674 \pm 0.118$ & $20.065 \pm 0.065$ & $19.026 \pm 0.038$ & $18.012 \pm 0.017$ & $17.484 \pm 0.010$ & $17.224 \pm 0.004$ & $15.629 \pm 0.0$ \\
\hline
\end{tabular}

Note. All HST photometric measurements are in the Vega system and have been obtained from data for this project. Magnitudes in F606W and F621M are from observations obtained with spatial scanning data; other measurements are from $2 \times 2$ binned data.

Star 18 (black) overlaid with the best-fitting model according to MKCLASS (red).

Because it can be difficult to estimate extinction along a line of sight at very low Galactic latitude, and because our up to 14band stellar photometry spanning $0.275-4.5 \mu \mathrm{m}$ can aid the determination, we started with a weaker prior having a Gaussian width of $0.5 \mathrm{mag}$, and then, on the basis of the a posteriori extinction estimates, we applied a global correction to the 2MASS estimates before reverting to the $0.3 \mathrm{mag}$ uncertainty for a final estimate. The maximum extinction we allowed in the fits was 1.2 times the total extinction to infinity along this line of sight estimated by Schlafly \& Finkbeiner (2011), which in turn was based on a rescaling of the IRASbased estimate of Finkbeiner et al. (1998). The scatter of the a posteriori extinction estimates for each star around the extinction prior at its (spectrophotometrically) estimated distance was $0.21 \mathrm{mag}$, with extinction values ranging from $A_{V} \approx 0$ at distance modulus $\mu=9$ mag to $A_{V}=3 \mathrm{mag}$ at $\mu=13$ mag. Figure 12 shows the resulting final law for $A_{V}$ versus $\mu$ (red points and red line), together with the individual values for each of the reference stars in the field (blue squares). The relation thus obtained between extinction and distance, for stars both closer by and farther away than the Cepheid, will also serve to constrain the reddening estimated for the Cepheid. This will in turn provide information on the intrinsic colors of the Cepheids and improve the robustness of the global $P-L$ calibration we expect to obtain.

\section{THE ABSOLUTE PARALLAX OF SS CMA}

\subsection{Multi-epoch Combination and Parallax Fit}

The final step in the astrometric solution consists of combining the multiple measurement epochs taken over the course of $2 \mathrm{yr}$ at intervals of 6 months to fit to our standard astrometric model, which involves three parameters for each star: position, parallax, and proper motion along the measurement direction. The results we present here for SS CMa are based on five epochs of observation; four more epochs are being obtained as part of a recently approved program extension (Program GO-14206). The fifth epoch does not include the serpentine scan. The exposures used are listed in Table 5.

Together with the astrometric parameters of each star, the model includes up to second-order geometric parameters used to align each epoch with one another (offset and rotation), as well as any residual large-scale adjustment to the geometric distortion required to reduce the model residuals. This last part is identical to the single-epoch aggregation step, but it now substitutes the stationary-star assumption with the astrometric model for each star.

The full model can be formally described by the expression

$$
\begin{aligned}
X_{i j}= & X_{i 0}-X_{\text {ref }, j}+P M x_{i}\left(t_{j}-t_{0}\right)+\pi_{i} f_{j} \\
& +R_{j} Y_{i 0}+\left\langle P_{j}\left(X_{\text {det }}, Y_{\text {det }}\right)\right\rangle_{\text {trail }_{i}},
\end{aligned}
$$


Table 3

Properties of the Reference Stars. II. Infrared Photometry

\begin{tabular}{|c|c|c|c|c|c|c|}
\hline Star & $\begin{array}{c}J \\
\text { 2MASS }\end{array}$ & $\begin{array}{c}\text { F160W } \\
\text { WFC3/IR }\end{array}$ & $\begin{array}{c}H \\
\text { 2MASS }\end{array}$ & $\begin{array}{c}K \\
\text { 2MASS }\end{array}$ & $\begin{array}{c}\text { Channel } 1 \\
\text { WISE }\end{array}$ & $\begin{array}{c}\text { Channel } 2 \\
\text { WISE }\end{array}$ \\
\hline 3 & $11.261 \pm 0.022$ & $\cdots$ & $10.642 \pm 0.021$ & $10.439 \pm 0.023$ & $10.282 \pm 0.026$ & $10.322 \pm 0.026$ \\
\hline 5 & $11.902 \pm 0.022$ & $\ldots$ & $11.813 \pm 0.022$ & $11.802 \pm 0.024$ & $\ldots$ & $\ldots$ \\
\hline 6 & $14.882 \pm 0.042$ & $\ldots$ & $14.375 \pm 0.064$ & $14.478 \pm 0.094$ & $\ldots$ & $\ldots$ \\
\hline 9 & $13.575 \pm 0.026$ & $\ldots$ & $13.099 \pm 0.021$ & $12.996 \pm 0.036$ & $\ldots$ & $\ldots$ \\
\hline 10 & $14.435 \pm 0.027$ & $\cdots$ & $13.960 \pm 0.043$ & $13.976 \pm 0.058$ & $13.880 \pm 0.030$ & $13.957 \pm 0.047$ \\
\hline 11 & $14.573 \pm 0.026$ & $\cdots$ & $14.173 \pm 0.035$ & $14.014 \pm 0.063$ & $13.829 \pm 0.031$ & $14.040 \pm 0.053$ \\
\hline 12 & $14.959 \pm 0.059$ & $\ldots$ & $14.374 \pm 0.065$ & $\ldots$ & $13.960 \pm 0.032$ & $14.049 \pm 0.051$ \\
\hline 14 & $11.646 \pm 0.024$ & $\ldots$ & $11.475 \pm 0.021$ & $11.455 \pm 0.024$ & $11.358 \pm 0.023$ & $\ldots$ \\
\hline 18 & $13.365 \pm 0.022$ & $\ldots$ & $13.162 \pm 0.034$ & $13.093 \pm 0.034$ & $\ldots$ & $\ldots$ \\
\hline 21 & $14.342 \pm 0.024$ & $14.125 \pm 0.017$ & $14.056 \pm 0.047$ & $13.877 \pm 0.059$ & $\ldots$ & $\ldots$ \\
\hline 23 & $\ldots$ & $14.840 \pm 0.023$ & $\ldots$ & $\ldots$ & $\ldots$ & $\ldots$ \\
\hline 25 & $12.914 \pm 0.024$ & $12.581 \pm 0.008$ & $12.514 \pm 0.024$ & $12.417 \pm 0.029$ & $\ldots$ & $\cdots$ \\
\hline 26 & $12.110 \pm 0.021$ & $11.935 \pm 0.006$ & $11.922 \pm 0.024$ & $11.761 \pm 0.023$ & $\ldots$ & $\ldots$ \\
\hline 29 & $\ldots$ & $\ldots$ & $\ldots$ & $\ldots$ & $\ldots$ & $\ldots$ \\
\hline 31 & $13.759 \pm 0.021$ & $13.417 \pm 0.012$ & $13.326 \pm 0.022$ & $13.282 \pm 0.036$ & $\ldots$ & $\ldots$ \\
\hline 37 & $9.453 \pm 0.022$ & $\ldots$ & $8.703 \pm 0.061$ & $8.481 \pm 0.019$ & $\ldots$ & $\ldots$ \\
\hline 38 & $13.402 \pm 0.024$ & $12.993 \pm 0.010$ & $12.876 \pm 0.022$ & $12.782 \pm 0.027$ & $\ldots$ & $\ldots$ \\
\hline 39 & $14.910 \pm 0.047$ & $\ldots$ & $14.395 \pm 0.044$ & $14.219 \pm 0.073$ & $\cdots$ & $\ldots$ \\
\hline 43 & $11.741 \pm 0.030$ & $\ldots$ & $10.762 \pm 0.028$ & $10.492 \pm 0.024$ & $\ldots$ & $10.336 \pm 0.022$ \\
\hline 44 & $\ldots$ & $14.449 \pm 0.015$ & $\cdots$ & $\ldots$ & $\ldots$ & $\ldots$ \\
\hline 45 & $12.555 \pm 0.022$ & $\cdots$ & $11.749 \pm 0.026$ & $11.486 \pm 0.021$ & $\ldots$ & $11.406 \pm 0.023$ \\
\hline 66 & $\cdots$ & $14.484 \pm 0.020$ & $\cdots$ & $\cdots$ & $\cdots$ & $\cdots$ \\
\hline $\mathrm{n} 1$ & $15.631 \pm 0.062$ & $\ldots$ & $15.543 \pm 0.118$ & $15.515 \pm 0.216$ & $15.014 \pm 0.042$ & $\cdots$ \\
\hline $\mathrm{n} 2$ & $15.663 \pm 0.055$ & $\ldots$ & $15.020 \pm 0.059$ & $\ldots$ & $\cdots$ & $\cdots$ \\
\hline $\mathrm{n} 4$ & $14.480 \pm 0.036$ & $13.879 \pm 0.015$ & $13.704 \pm 0.026$ & $13.475 \pm 0.035$ & $\ldots$ & $\ldots$ \\
\hline $\mathrm{n} 7$ & $15.319 \pm 0.037$ & $14.901 \pm 0.024$ & $14.802 \pm 0.047$ & $\ldots$ & $\ldots$ & $\cdots$ \\
\hline n10 & $14.145 \pm 0.035$ & $\ldots$ & $13.400 \pm 0.029$ & $13.119 \pm 0.033$ & $\ldots$ & $\ldots$ \\
\hline $\mathrm{n} 11$ & $14.671 \pm 0.027$ & $\ldots$ & $13.888 \pm 0.038$ & $13.780 \pm 0.046$ & $\ldots$ & $\ldots$ \\
\hline
\end{tabular}

Note. All HST photometric measurements are in the Vega system and have been obtained from data for this project. Photometry obtained from 2MASS (Skrutskie et al. 2006) and from WISE (Wright et al. 2010) are in their respective systems.

where the basic measurements are the positions $X_{i j}$ that is, the $X$ position of the trail of star $i$ in image $j$ (relative to the reference scan line)-measured after correction for variable rotation, scale-corrected for velocity aberration and variable distortion, and projected onto a constant sky frame. The $X$ coordinate is aligned with detector $X$ and, by design, aligned with the bulk of the parallactic motion. The quantity $X_{i 0}$ is the reference position of star $i$ at time $t_{0}$, and $X_{\text {ref, } j}$ is the offset of image $j$ in the $X$ direction-in essence, the position of the reference scan line for image $j$ on the sky. The astrometric motion of star $i$ in the $X$ direction is described by the $X$ component of the proper motion, $P M x_{i}$, and the parallax $\pi_{i}$, applied with the epoch-dependent parallax factor $f_{j}$. The term $f_{j}$ is the projection (for unit parallax) of the parallactic motion in the $X$ direction at the time of the observations, computed using the formulae on pp. B28 and C5 of The Astronomical Almanac (2013) and the orientation of the detector axes from the image headers.

As we did for SY Aur, the model position must be corrected for the relative rotation and geometric distortion of image $j$ with respect to the reference image. The rotation term on the sky is $R_{j} Y_{i 0}$, where $R_{j}$ is the rotation of image $j$ and $Y_{i 0}$ is the static relative position of star $i$ in rectified coordinates along the $Y$ direction with respect to the center of the field. We find our rotations to be of order $10^{-5}$, so even a coarse measurement of $Y_{i 0}$ with a precision of $\sim 1$ pixel will suffice. The polynomial term is determined simultaneously with the astrometric parameters during the model-fitting procedure, as a seconddegree $P_{j}\left(X_{\mathrm{det}}, Y_{\mathrm{det}}\right)$, where $X_{\mathrm{det}}$ and $Y_{\mathrm{det}}$ are detector coordinates; the total correction is determined by evaluating the polynomial for image $j$ at every location along the trail of star $i$ in that image and averaging the result. The constant term is omitted from the polynomial because it is degenerate with the image offset $X_{\text {ref }, j}$.

Our proper-motion term is relative to the set of stars in the field and contains a contribution from the estimated X-CTE term per year at that star's location.

Note also that the model is formulated to be linear in the astrometric parameters, which in turn are linearly related to most measured quantities, i.e., positions on the detector. As a consequence, the errors in the derived astrometric parameters for the Cepheid are likely to be very nearly Gaussian (the same does not necessarily apply to distant stars for which the spectrophotometric constraints dominate the error distribution). As long as the parallax and its error distribution are used directly, there is no need to apply nonlinear corrections such as those suggested by Lutz \& Kelker (1973). More generally, proper consideration of all prior information used in selecting and characterizing the population of our target Cepheids will be required in determining the optimal calibration for the $P-L$ relation on the basis of our measurements (Hanson 1979; Francis 2013; see also the discussion in Benedict et al. 2007). For SS CMa, the distance modulus bias is most likely smaller than for most Cepheids in the Benedict et al. (2007) sample, because of the different geometry; we currently estimate that the correction is $-0.03 \pm 0.02 \mathrm{mag}$, based on its Galactic 
Table 4

Properties of the Reference Stars. III. Spectra, Classification, and Astrometry

\begin{tabular}{|c|c|c|c|c|c|c|c|c|}
\hline Star & $\begin{array}{l}\text { R.A. (deg) } \\
\text { (J2000) }\end{array}$ & $\begin{array}{l}\text { Decl. (deg) } \\
\text { (J2000) }\end{array}$ & $\begin{array}{l}\text { Spectrum } \\
\text { Source }\end{array}$ & Class $^{\mathrm{a}}$ & Quality $^{\mathrm{b}}$ & $\begin{array}{l}T_{\text {eff }}{ }^{\mathrm{c}} \\
(\mathrm{K})\end{array}$ & $\begin{array}{l}\pi_{\text {full }}{ }^{\mathrm{d}} \\
\text { (mas) }\end{array}$ & $\begin{array}{l}\pi_{\text {astro }}{ }^{\mathrm{e}} \\
\text { (mas) }\end{array}$ \\
\hline 3 & 111.49716 & -25.23251 & Gemini & K0 IV & $\mathrm{F}$ & $5340 \pm 160$ & $0.414 \pm 0.043$ & $\overline{0.166 \pm 0.110}$ \\
\hline 5 & 111.51980 & -25.24066 & Gemini & A7 V & G & $7920 \pm 250$ & $1.185 \pm 0.049$ & $1.197 \pm 0.053$ \\
\hline 9 & 111.53022 & -25.23712 & Lick & G0 IV-V & VG & $6180 \pm 180$ & $\ldots$ & $\cdots-$ \\
\hline 10 & 111.54177 & -25.23063 & Gemini & F7 IV & VG & $6550 \pm 200$ & $0.448 \pm 0.096$ & $0.060 \pm 0.260$ \\
\hline 11 & 111.52409 & -25.22336 & Lick & F8 III & $\mathrm{F}$ & $6420 \pm 160$ & $\cdots$ & $\cdots-$ \\
\hline 18 & 111.51415 & -25.25188 & Lick & $\mathrm{B} 2 \mathrm{~V}$ & $\mathrm{~F}$ & $21000 \pm 1000$ & $0.281 \pm 0.023$ & $0.339 \pm 0.041$ \\
\hline 21 & 111.53000 & -25.26841 & Gemini & A4 III-IV & VG & $8400 \pm 340$ & $0.333 \pm 0.027$ & $0.297 \pm 0.036$ \\
\hline 23 & 111.53011 & -25.26374 & Gemini & F8 V & VG & $6420 \pm 160$ & $0.398 \pm 0.036$ & $0.386 \pm 0.040$ \\
\hline 25 & 111.54306 & -25.27473 & Lick & G5 IV-V & G & $5730 \pm 110$ & $0.688 \pm 0.037$ & $0.683 \pm 0.040$ \\
\hline 26 & 111.52272 & -25.28065 & Lick & A2 III-IV & VG & $8680 \pm 360$ & $\cdots$ & $\cdots-$ \\
\hline 29 & 111.53344 & -25.25502 & Gemini & G6 V & VG & $5690 \pm 130$ & $1.012 \pm 0.038$ & $1.023 \pm 0.041$ \\
\hline 43 & 111.52175 & -25.28641 & Keck & K6 Iab & G & $5500 \pm 260$ & $0.032 \pm 0.008$ & $0.018 \pm 0.064$ \\
\hline 44 & 111.52294 & -25.28780 & Lick & G2 IV-V & G & $5800 \pm 180$ & $0.797 \pm 0.037$ & $0.799 \pm 0.039$ \\
\hline 45 & 111.53057 & -25.28724 & Lick & F8 II-III & $\mathrm{F}$ & $6300 \pm 130$ & $0.257 \pm 0.022$ & $0.336 \pm 0.053$ \\
\hline 66 & 111.55038 & -25.26568 & Lick & G2 III & G & $5620 \pm 180$ & $0.366 \pm 0.040$ & $0.337 \pm 0.071$ \\
\hline $\mathrm{n} 1$ & 111.50797 & -25.23456 & Lick & $B 9^{f}$ & $\mathrm{P}$ & $\ldots$ & $0.548 \pm 0.063$ & $0.548 \pm 0.074$ \\
\hline n2 & 111.51711 & -25.26131 & Keck & F7 III-IV & G & $6480 \pm 200$ & $\cdots$ & $\cdots-$ \\
\hline $\mathrm{n} 4$ & 111.52766 & -25.26668 & Keck & $G 5^{f}$ & $\mathrm{~F}$ & $5660 \pm 110$ & $0.240 \pm 0.024$ & $0.124 \pm 0.043$ \\
\hline $\mathrm{n} 7$ & 111.54059 & -25.26129 & Keck & F7 V & G & $6480 \pm 200$ & $\cdots$ & $\cdots-$ \\
\hline $\mathrm{n} 10$ & 111.55420 & -25.25736 & Keck & $G 5^{f}$ & G & $5660 \pm 110$ & $\ldots$ & $\cdots-$ \\
\hline $\mathrm{n} 11$ & 111.50804 & -25.25449 & Keck & G9 IV-V & G & $5390 \pm 160$ & $0.331 \pm 0.036$ & $0.376 \pm 0.060$ \\
\hline
\end{tabular}

Notes.

a Spectral classification based on MKCLASS Version 1.7 (Gray \& Corbally 2014; see text for details) unless otherwise noted.

${ }^{\mathrm{b}}$ Classification quality from MKCLASS; VG = Very good, $\mathrm{G}=\mathrm{Good}, \mathrm{F}=$ Fair, $\mathrm{P}=$ Poor.

c Temperature and uncertainty from the spectroscopic analysis.

d Parallax estimate based on combined spectrophotometric and astrometric data.

e Parallax estimate based purely on astrometric data for each star; spectrophotometric information is retained for all other stars. See Section 4.2 for details.

${ }^{\mathrm{f}}$ Classification and quality from match to model spectra; luminosity class not available.

latitude and distance. Therefore, we do not include a LutzKelker-type correction for the parallax of SS CMa at this point; full consideration of the distance probability distribution of for each Cepheid, taking into account both selection and observational biases, will be included in the analysis of the full sample of Cepheids.

As far as the astrometric model is concerned, parallaxes are also relative; however, the degeneracy in the conversion to absolute parallaxes can be broken by using the spectrophotometric distance estimates for the stars in the field discussed in Section 3. The distance estimate of the target star will be insensitive to uncertainties in the distance of the reference stars so long as the set contains objects that are bright and distant (e.g., red giants). Note also that in addition to providing a conversion to absolute parallax, individual spectrophotometric parallaxes are also helpful in constraining some epoch-to-epoch geometric transformations. We will discuss in detail in Section 4.2 the impact of the parallax constraints for reference stars on the multi-epoch solution and investigate the consequences of uncertainties, outliers, and other possible issues.

Each epoch after the first is allowed a net offset and a second-degree polynomial adjustment to match the first epoch; since there are about 20 stars useful for measurement at each epoch, these additional six parameters per epoch over which we marginalize do not place an undue burden on the solution.

Formally, the a priori distance estimates based on spectrophotometric parallaxes serve as Bayesian priors for the parallax of the stars in the field. A prior is not used for the Cepheid, so that its distance estimate is determined directly and only from its observed parallax.

The best values of the model parameters are determined by minimizing the total model $\chi^{2}$ to achieve the most likely parameters. Among these parameters is the absolute parallax of SS CMa, which results directly from the model optimization. A modest fraction of the reference stars in the field are expected to be part of binaries with parameters that would cause a significant deviation from our simple astrometric model. This fraction depends on distance and spectral class, but is $\sim 10 \%-$ $20 \%$ for $\mathrm{F}$ and $\mathrm{G}$ stars at $1 \mathrm{kpc}$ on the basis of the distribution of binary properties in Duquennoy \& Mayor (1991; see also discussion in Paper I). We run the global model iteratively after rejecting one outlier (Star n2) on the basis of its disproportionate contribution to the total $\chi^{2}$. We also exclude Star n10 because its astrometric parallax is suspect, resulting in a very 


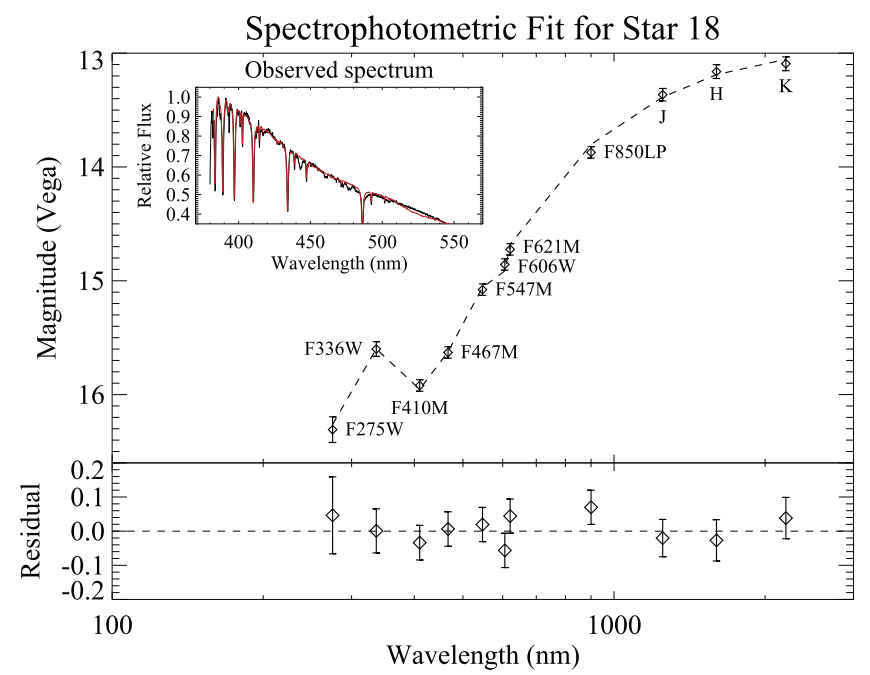

Figure 11. Spectral and photometric fit for the B2 V star 18. Top panel: observed photometry (diamonds) and best-fitting model from the Padova isochrones (dashed line). Inset: observed spectrum (black), continuumcorrected and fitted to a model spectrum (red) using MKCLASS. Bottom panel: residuals of the model photometric fit, shown on a larger vertical scale. Photometric errors include a $0.05 \mathrm{mag}$ term added in quadrature to the measurement uncertainty to account for possible differences in photometric systems between models and observations.

\section{Extinction (prior and fitted) for the SS CMa field}

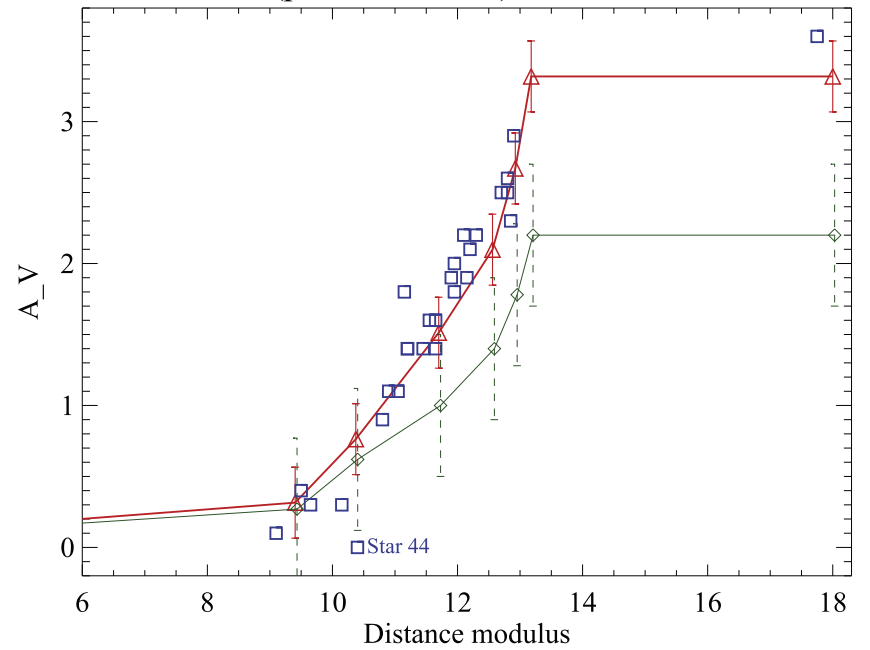

Figure 12. Estimated extinction vs. distance modulus for the SS CMa field. The green curve and values (diamonds with error bars) show the starting estimate of the relation between extinction and distance, which is iteratively adjusted during the fit process. The red curve and values (open triangles with error bars) show the final relation. The blue squares show the estimated extinction for each of the reference stars with sufficient spectrophotometric information.

large estimated distance, which is inconsistent with its spectrophotometric information.

\subsubsection{Multi-parametric Model for SS CMa: Primary Solution}

Figure 13 shows the best estimate of the parallax for the stars in the SS CMa field. For reference stars, the reported parallax combines both astrometric and spectrophotometric information; no spectrophotometric information is used for the Cepheid SS CMa (the star labeled 0).

For each star, the top panel shows the astrometric measurements at each epoch (dots with error bars) and the best-fitting parallax model (red line), both in milliarcseconds; the fitted proper motion is subtracted from both the measurements and the models for ease of display. The gray band shows the spectrophotometric parallax estimate with a $2 \sigma$ uncertainty, when available. The bottom panel shows the astrometric residuals from the best model, also in milliarcseconds.

The best estimate of the parallax of SS CMa is $0.348 \pm$ 0.038 mas, corresponding to a distance estimate of $2.87 \pm$ $0.33 \mathrm{kpc}$. However, note that, as commented in Section 4.1, the error distribution is likely Gaussian only in parallax. A nonlinear conversion, e.g., to distance, will have a nonsymmetric error distribution, which must be taken into account in further processing. It is also necessary to consider any prior information used in selecting and characterizing the sample (Hanson 1979; Francis 2013; see also the analysis in Benedict et al. 2007).

The uncertainty in the conversion to absolute parallax (i.e., the systematic uncertainty in the frame parallax) of the set of 20 fitted reference stars is $7 \mu$ as, well below our target uncertainty. In the field of SS CMa, the precision of the conversion to absolute parallax benefits from the presence of some very distant stars, and especially Star 43, whose spectrophotometry indicates that it is a $\mathrm{K}$ giant at around $30 \mathrm{kpc}$ (Section 4.3). However, even excluding Star 43, the rest of the reference stars indicate an uncertainty in the frame parallax of about $11 \mu \mathrm{as}$, still much smaller than our target uncertainty. This is not surprising; Figure 14 shows the typical precision of the correction to absolute parallax in random fields generated from the Besançon model for the direction of SS CMa, assuming a $15 \%$ typical uncertainty in spectrophotometric distance estimates and that only half of the stars in the field will be available for the conversion. A typical field would have 20 available reference stars and an uncertainty of $9 \mu$ as in the conversion to absolute parallax for an assumed rms uncertainty of 0.3 mag in the estimated distance moduli, comparable to the values for the actual data. (The uncertainty in the conversion to absolute parallax will scale roughly linearly with the assumed uncertainty in the distance moduli.) However, Star 43 is unusual in its own right, and it will be further discussed in Section 4.2.

\subsection{Astrometric versus Spectrophotometric Parallax: Partially Constrained Solutions}

In the primary solution, we assumed that the spectrophotometric and astrometric distance estimates for each star are separate but valid measurements of the same quantity, the physical distance of each star. In practice, this assumption may not always be true, e.g., because of the possible binarity discussed earlier. On the other hand, the assumptions underlying our spectrophotometric distance estimates may not be valid for all stars, and there could be outliers-e.g., due to anomalous extinction, or a history of mass exchange-which could occasionally lead to a faulty estimate of the distance modulus.

A careful comparison of astrometric and spectrophotometric parallaxes for the reference stars can provide a powerful check of our procedures and our final accuracy. Spectrophotometric parallaxes, derived from a combination of spectra and multiband photometry in conjunction with stellar model tracks, a model of the density distribution of stars along the line of sight, and an extinction model, typically have fractional accuracy that varies little as a function of distance; thus, their absolute error is 
Table 5

Spatial Scanning Observations Used in This Paper

\begin{tabular}{|c|c|c|c|c|c|c|c|c|c|}
\hline Date & Rootname & Program ID & $\begin{array}{l}\text { EXPSTART } \\
\text { (MJD) }\end{array}$ & Filter & $\begin{array}{l}\text { Exp. time } \\
\text { (s) }\end{array}$ & $\begin{array}{l}\text { Scan Rate } \\
\left(\operatorname{arcsec~s}^{-1}\right)\end{array}$ & $\begin{array}{l}\text { Scan Length } \\
(\operatorname{arcsec})\end{array}$ & $\begin{array}{l}\text { Number } \\
\text { of Legs }\end{array}$ & $\begin{array}{l}X \text { pos targ } \\
(\operatorname{arcsec})\end{array}$ \\
\hline \multicolumn{10}{|c|}{ Observations for SS CMa } \\
\hline 2012 Oct 23 & ibzc04kjq & 12879 & 56223.905323 & F606W & 350.0 & 0.330 & 115.5 & 1 & -3.00 \\
\hline 2012 Oct 23 & ibzc04klq & 12879 & 56223.911133 & F621M & 350.0 & 0.330 & 115.5 & 1 & -3.00 \\
\hline 2012 Oct 23 & ibzc04knq & 12879 & 56223.916712 & F621M & 350.0 & 0.330 & 115.5 & 1 & -3.00 \\
\hline 2012 Oct 23 & ibzc04kpq & 12879 & 56223.922592 & F606W & 350.0 & 0.330 & 115.5 & 1 & -3.00 \\
\hline 2012 Oct 23 & ibzc04krq & 12879 & 56223.928899 & F606W & 350.0 & 1.505 & 526.7 & 3 & -10.00 \\
\hline 2013 Apr 18 & ibzc15ntq & 12879 & 56400.332005 & F606W & 348.0 & 0.330 & 114.8 & 1 & -3.00 \\
\hline 2013 Apr 18 & ibzc15nvq & 12879 & 56400.337792 & F621M & 348.0 & 0.330 & 114.8 & 1 & -3.00 \\
\hline 2013 Apr 18 & ibzc15nxq & 12879 & 56400.343347 & F621M & 348.0 & 0.330 & 114.8 & 1 & -3.00 \\
\hline 2013 Apr 18 & ibzc15o0q & 12879 & 56400.349204 & F606W & 348.0 & 0.330 & 114.8 & 1 & -3.00 \\
\hline 2013 Apr 18 & ibzc15o2q & 12879 & 56400.355489 & F606W & 348.0 & 1.505 & 523.7 & 3 & -10.00 \\
\hline 2013 Oct 22 & ic8z04haq & 13344 & 56587.580458 & F606W & 348.0 & 0.330 & 114.8 & 1 & -3.00 \\
\hline 2013 Oct 22 & ic8z04hcq & 13344 & 56587.586246 & F621M & 348.0 & 0.330 & 114.8 & 1 & -3.00 \\
\hline 2013 Oct 22 & ic8z04heq & 13344 & 56587.591801 & F621M & 348.0 & 0.330 & 114.8 & 1 & -3.00 \\
\hline 2013 Oct 22 & ic8z04hgq & 13344 & 56587.597657 & F606W & 348.0 & 0.330 & 114.8 & 1 & -3.00 \\
\hline 2013 Oct 22 & ic8z04hiq & 13344 & 56587.603942 & F606W & 348.0 & 1.505 & 523.7 & 3 & -10.00 \\
\hline 2014 Apr 16 & ic8z15o4q & 13344 & 56763.310662 & F606W & 348.0 & 0.330 & 114.8 & 1 & +3.00 \\
\hline 2014 Apr 16 & ic8z15o6q & 13344 & 56763.316449 & F621M & 348.0 & 0.330 & 114.8 & 1 & +3.00 \\
\hline 2014 Apr 16 & ic8z15o8q & 13344 & 56763.322005 & F621M & 348.0 & 0.330 & 114.8 & 1 & +3.00 \\
\hline 2014 Apr 16 & ic8z15oaq & 13344 & 56763.327862 & F606W & 348.0 & 0.330 & 114.8 & 1 & +3.00 \\
\hline 2014 Apr 16 & ic8z15ocq & 13344 & 56763.334146 & F606W & 348.0 & 1.505 & 523.7 & 3 & +2.00 \\
\hline 2014 Oct 23 & icir03ixq & 13678 & 56953.729151 & F606W & 348.0 & 0.330 & 114.8 & 1 & -3.00 \\
\hline 2014 Oct 23 & icir03j3q & 13678 & 56953.746350 & F606W & 348.0 & 0.330 & 114.8 & 1 & -3.00 \\
\hline 2014 Oct 23 & icir03izq & 13678 & 56953.734938 & F621M & 348.0 & 0.330 & 114.8 & 1 & -3.00 \\
\hline 2014 Oct 23 & icir03j1q & 13678 & 56953.740494 & F621M & 348.0 & 0.330 & 114.8 & 1 & -3.00 \\
\hline \multicolumn{10}{|c|}{ Observations for M48 } \\
\hline 2014 Sep 25 & icmp04ptq & 13929 & 56925.069671 & F673N & 348.0 & 0.400 & 139.2 & 1 & -6.90 \\
\hline 2014 Sep 25 & icmp04pvq & 13929 & 56925.075678 & F673N & 348.0 & 0.400 & 139.2 & 1 & -33.10 \\
\hline 2014 Sep 25 & icmp04pyq & 13929 & 56925.081812 & F673N & 348.0 & 0.400 & 139.2 & 1 & +26.20 \\
\hline 2014 Sep 25 & icmp04q0q & 13929 & 56925.087738 & F673N & 348.0 & 0.400 & 139.2 & 1 & +14.40 \\
\hline 2014 Sep 25 & icmp04q2q & 13929 & 56925.131662 & F673N & 348.0 & 0.400 & 139.2 & 1 & +43.50 \\
\hline 2014 Sep 25 & icmp06qnq & 13929 & 56925.274092 & F673N & 348.0 & 0.400 & 139.2 & 1 & +8.50 \\
\hline 2014 Sep 25 & icmp06qpq & 13929 & 56925.280227 & F673N & 348.0 & 0.400 & 139.2 & 1 & +57.50 \\
\hline 2014 Sep 25 & icmp06qrq & 13929 & 56925.286199 & F673N & 348.0 & 0.400 & 139.2 & 1 & -12.00 \\
\hline 2014 Sep 25 & icmp06qtq & 13929 & 56925.337796 & F673N & 348.0 & 0.400 & 139.2 & 1 & +17.40 \\
\hline 2014 Sep 25 & icmp06qvq & 13929 & 56925.343907 & F673N & 348.0 & 0.400 & 139.2 & 1 & -24.00 \\
\hline 2014 Sep 28 & icmp05xlq & 13929 & 56928.863641 & F673N & 348.0 & 0.400 & 139.2 & 1 & -21.20 \\
\hline 2014 Sep 28 & icmp05xnq & 13929 & 56928.869741 & F673N & 348.0 & 0.400 & 139.2 & 1 & +36.30 \\
\hline 2014 Sep 28 & icmp05xpq & 13929 & 56928.907391 & F673N & 348.0 & 0.400 & 139.2 & 1 & +16.30 \\
\hline 2014 Sep 28 & icmp05xrq & 13929 & 56928.913410 & F673N & 348.0 & 0.400 & 139.2 & 1 & -47.20 \\
\hline 2014 Sep 28 & icmp05xtq & 13929 & 56928.919486 & F673N & 348.0 & 0.400 & 139.2 & 1 & +3.30 \\
\hline 2014 Sep 29 & icmp01gmq & 13929 & 56929.974255 & F621M & 348.0 & 0.400 & 139.2 & 1 & -6.90 \\
\hline 2014 Sep 29 & icmp01goq & 13929 & 56929.980261 & F621M & 348.0 & 0.400 & 139.2 & 1 & -33.10 \\
\hline 2014 Sep 29 & icmp01gqq & 13929 & 56929.986396 & F621M & 348.0 & 0.400 & 139.2 & 1 & +26.20 \\
\hline 2014 Sep 29 & icmp01gsq & 13929 & 56929.992322 & F621M & 348.0 & 0.400 & 139.2 & 1 & +14.40 \\
\hline 2014 Sep 29 & icmp01guq & 13929 & 56929.998340 & F621M & 348.0 & 0.400 & 139.2 & 1 & +43.50 \\
\hline 2014 Sep 30 & icmp03h5q & 13929 & 56930.039567 & F621M & 348.0 & 0.400 & 139.2 & 1 & +8.50 \\
\hline 2014 Sep 30 & icmp03haq & 13929 & 56930.045701 & F621M & 348.0 & 0.400 & 139.2 & 1 & +57.50 \\
\hline 2014 Sep 30 & icmp03hcq & 13929 & 56930.051674 & F621M & 348.0 & 0.400 & 139.2 & 1 & -12.00 \\
\hline 2014 Sep 30 & icmp03hfq & 13929 & 56930.057819 & F621M & 348.0 & 0.400 & 139.2 & 1 & +17.40 \\
\hline 2014 Sep 30 & icmp03hhq & 13929 & 56930.063931 & F621M & 348.0 & 0.400 & 139.2 & 1 & -24.00 \\
\hline 2014 Oct 01 & icmp02odq & 13929 & 56931.174394 & F621M & 348.0 & 0.400 & 139.2 & 1 & -21.20 \\
\hline 2014 Oct 01 & icmp02ofq & 13929 & 56931.180493 & F621M & 348.0 & 0.400 & 139.2 & 1 & +36.30 \\
\hline 2014 Oct 01 & icmp02ohq & 13929 & 56931.186662 & F621M & 348.0 & 0.400 & 139.2 & 1 & +16.30 \\
\hline 2014 Oct 01 & icmp02ojq & 13929 & 56931.192681 & F621M & 348.0 & 0.400 & 139.2 & 1 & -47.20 \\
\hline 2014 Oct 01 & icmp02opq & 13929 & 56931.236743 & F621M & 348.0 & 0.400 & 139.2 & 1 & +3.30 \\
\hline
\end{tabular}


Table 5

(Continued)

\begin{tabular}{|c|c|c|c|c|c|c|c|c|c|}
\hline Date & Rootname & Program ID & $\begin{array}{l}\text { EXPSTART } \\
\text { (MJD) }\end{array}$ & Filter & $\begin{array}{l}\text { Exp. time } \\
\text { (s) }\end{array}$ & $\begin{array}{l}\text { Scan Rate } \\
\left(\operatorname{arcsec~s}^{-1}\right)\end{array}$ & $\begin{array}{l}\text { Scan Length } \\
(\operatorname{arcsec})\end{array}$ & $\begin{array}{l}\text { Number } \\
\text { of Legs }\end{array}$ & $\begin{array}{c}X \text { pos targ } \\
(\operatorname{arcsec})\end{array}$ \\
\hline \multicolumn{10}{|c|}{ Observations for M67 } \\
\hline 2014 Nov 08 & icmp07m8q & 13929 & 56969.071721 & F606W & 348.0 & 0.400 & 139.2 & 1 & -30.34 \\
\hline 2014 Nov 08 & icmp07maq & 13929 & 56969.077670 & F606W & 348.0 & 0.400 & 139.2 & 1 & -14.35 \\
\hline 2014 Nov 08 & icmp07mdq & 13929 & 56969.083758 & F606W & 348.0 & 0.400 & 139.2 & 1 & +30.88 \\
\hline 2014 Nov 08 & icmp07mfq & 13929 & 56969.132601 & F606W & 348.0 & 0.400 & 139.2 & 1 & +36.42 \\
\hline 2014 Nov 08 & icmp07mhq & 13929 & 56969.138955 & F606W & 348.0 & 0.400 & 139.2 & 1 & +39.60 \\
\hline 2014 Nov 08 & icmp08nyq & 13929 & 56969.524129 & F606W & 350.0 & 0.400 & 140.0 & 1 & +7.05 \\
\hline 2014 Nov 08 & icmp08o0q & 13929 & 56969.530055 & F606W & 350.0 & 0.400 & 140.0 & 1 & -2.88 \\
\hline 2014 Nov 08 & icmp08o2q & 13929 & 56969.536003 & F606W & 350.0 & 0.400 & 140.0 & 1 & +8.96 \\
\hline 2014 Nov 08 & icmp08o4s & 13929 & 56969.541988 & F606W & 350.0 & 0.400 & 140.0 & 1 & +26.71 \\
\hline 2014 Nov 08 & icmp08o6s & 13929 & 56969.547902 & F606W & 350.0 & 0.400 & 140.0 & 1 & +18.54 \\
\hline 2014 Nov 08 & icmp10q6q & 13929 & 56969.922312 & F606W & 350.0 & 0.400 & 140.0 & 1 & +3.90 \\
\hline 2014 Nov 08 & icmp10q8q & 13929 & 56969.928295 & F606W & 350.0 & 0.400 & 140.0 & 1 & +0.28 \\
\hline 2014 Nov 08 & icmp10qaq & 13929 & 56969.934302 & F606W & 350.0 & 0.400 & 140.0 & 1 & +3.89 \\
\hline 2014 Nov 08 & icmp10qcq & 13929 & 56969.940321 & F606W & 350.0 & 0.400 & 140.0 & 1 & -16.75 \\
\hline 2014 Nov 08 & icmp10qeq & 13929 & 56969.946281 & F606W & 350.0 & 0.400 & 140.0 & 1 & -22.13 \\
\hline 2014 Nov 09 & icmp09vkq & 13929 & 56970.851154 & F606W & 350.0 & 0.400 & 140.0 & 1 & -5.14 \\
\hline 2014 Nov 09 & icmp09vmq & 13929 & 56970.857022 & F606W & 350.0 & 0.400 & 140.0 & 1 & +13.52 \\
\hline 2014 Nov 09 & icmp09voq & 13929 & 56970.862890 & F606W & 350.0 & 0.400 & 140.0 & 1 & -8.37 \\
\hline 2014 Nov 09 & icmp09vqq & 13929 & 56970.868886 & F606W & 350.0 & 0.400 & 140.0 & 1 & +15.69 \\
\hline 2014 Nov 09 & icmp09vsq & 13929 & 56970.874777 & F606W & 350.0 & 0.400 & 140.0 & 1 & +2.15 \\
\hline
\end{tabular}

much smaller for distant stars than for nearby ones. On the other hand, astrometric parallaxes have absolute errors that are similar in magnitude in terms of parallax angle, and hence nearly independent of distance, although they do scale with apparent brightness; thus, for nearby stars, astrometric parallaxes are more accurate than spectrophotometric ones, and vice versa for distant stars. For typical stars in our analysis, the two accuracies are comparable at about $1 \mathrm{kpc}$. Consequently, stars beyond $1 \mathrm{kpc}$ provide a solid check of astrometric measurements, while closer stars provide a verification of the spectrophotometric estimates.

However, the parallax estimated for each star in the full (primary) solution shown in Figure 13 is affected by its spectrophotometric prior and hence cannot be used directly for an independent check of the astrometric parallax thus obtained. On the other hand, dropping all of the spectrophotometric priors is not a viable option. First, all narrow-field parallax measurements are, by necessity, only relative; thus, without some spectrophotometric measurements, no absolute parallax can be derived. Second, as discussed in Section 4.1, without a spectrophotometric prior for the majority of the stars, we lack the ability to properly constrain the relative alignment and polynomial distortion for each epoch of observation, thus worsening the quality of the measurements and introducing substantial degeneracies in the solution process.

In order to carry out a meaningful test of the quality of our astrometric parallaxes, we repeat the multi-epoch fit by discarding the spectrophotometric prior for each star in turn, and we define the parallax obtained for that star as its pure astrometric parallax. For example, when measuring the pure astrometric parallax for Star 29, we discard the spectrophotometric prior only for Star 29, but retain the prior for all the other stars in the fields that have one. This allows the solution to converge with only a minor decrease in overall precision, resulting in a trigonometric parallax estimate to Star
29 that is completely independent of any photometric or spectroscopic information for that star. The pure astrometric parallax is listed as $\pi_{\text {astro }}$ in Table 4 , where the parallax resulting from the full solution is labeled $\pi_{\text {full }}$. We then repeat this process for all other stars for which a spectrophotometric prior is available; for stars without a spectrophotometric prior, including the Cepheid, the pure astrometric parallax is of course identical to the parallax from the full solution. This procedure not only allows us to assess the quality of our astrometric measurements with the spectrophotometric distance estimates but also mimics the handling of the Cepheid itself, for which no spectrophotometric prior is ever used, and thus provides a useful test of the validity of its parallax measurement.

Figure 15 shows a comparison of the pure astrometric and spectrophotometric parallaxes for the reference stars in the field of SS CMa. The Cepheid is not included, as no spectrophotometric prior is used for it. For the majority of the stars, there is a very reasonable agreement between them, with 17 out of 20 within nominal $2 \sigma$; only Stars 3, 38, and n4 are outside this range, and Star 10 has a very small value for the pure astrometric parallax, with a very large uncertainty.

Excluded from Figure 15 is Star n10, for which the pure astrometric parallax is negative. In our solution, "negative" parallaxes are not necessarily disallowed; they can occur, for example, if the correction to absolute parallax is underestimated, and all stars are in reality closer than the astrometric parallax indicates. In that case, the true parallax would simply be larger than the resulting value, because of the larger correction to absolute parallax. However, in this case the solid agreement between astrometric and spectrophotometric parallaxes for most stars argues strongly against a large systematic error in the reduction to absolute parallax. Although we could limit the solution to require positive parallaxes, this step would be somewhat arbitrary, given that the actual value of the 

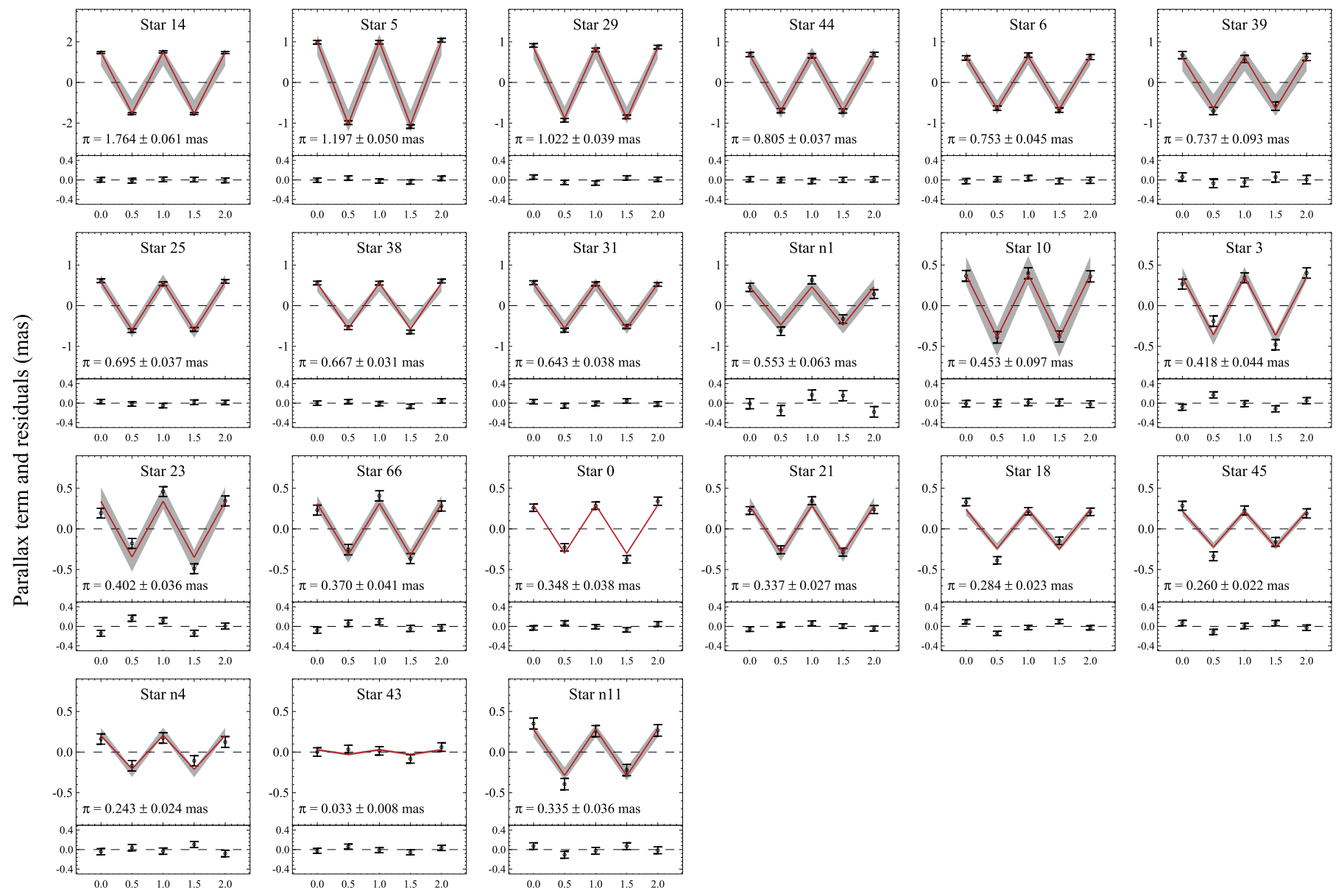

Epoch (years)

Figure 13. Individual stellar parallaxes in the field of SS CMa. The red line indicates the measured parallax; the gray band indicates the spectrophotometric parallax with $\pm 2 \sigma$ width. The Cepheid SS CMa is Star 0. Fitted proper motions have been subtracted from the measurements and fits for ease of viewing. Star 29, the putative companion of the Cepheid, is much closer to the Sun and is not physically associated with it.

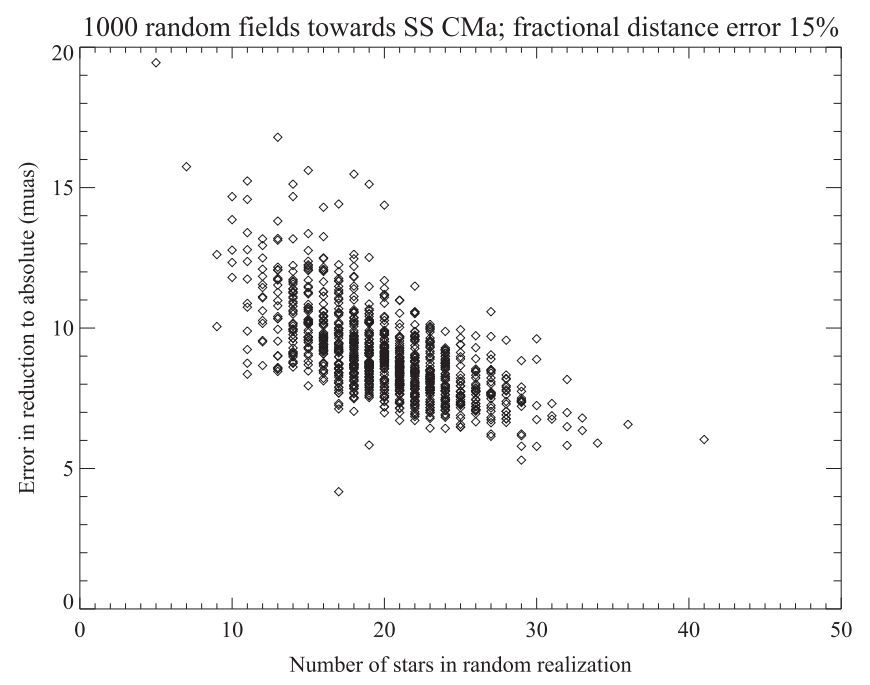

Figure 14. Distribution of expected errors in the conversion to absolute parallax for 1000 fields drawn randomly from the Besançon model in the direction of SS CMa. We assume that only half the stars in each field are available as reference stars, and that each has a $15 \%$ error in its spectrophotometric distance estimate (about $0.3 \mathrm{mag}$ in the estimated absolute magnitude). Typical values are $9 \mu$ as for $\sim 20$ stars, consistent with the actual values for our field when Star 43 is excluded. reference parallax is part of the optimization process, and could give excessive weight to stars with very low parallaxes in the solution. Therefore, we treat Star n10 as an astrometric outlier and exclude it from our solution. Based on the discussion in Paper I, we expect $\sim 10 \%$ of the reference stars to be astrometric outliers due to binarity, so the presence of an outlier in this sample is not surprising.

It is important to note that the accuracy of the partially constrained solutions obtained by dropping the spectrophotometric prior for one of the reference stars can be potentially compromised, especially if that star is near a corner of the field. The reason is that the low-order polynomial distortion that we adopt to register the measurements across epochs may lack a critical constraint near that corner, while on the other hand its value is required at that location. Consequently, a quasidegeneracy in the multi-epoch astrometric solution exists for that star. This is especially apparent for stars such as Star 3, which is the only bright star near the bottom left of the field (see Figure 3). The full solution (Figure 13) shows for Star 3 a normal parallax value and uncertainty of $0.418 \pm 0.044$ mas, and its residuals are fairly typical. On the other hand, the partially constrained solution in which its parallax prior is dropped is $0.167 \pm 0.111$ mas, with a very different value and 


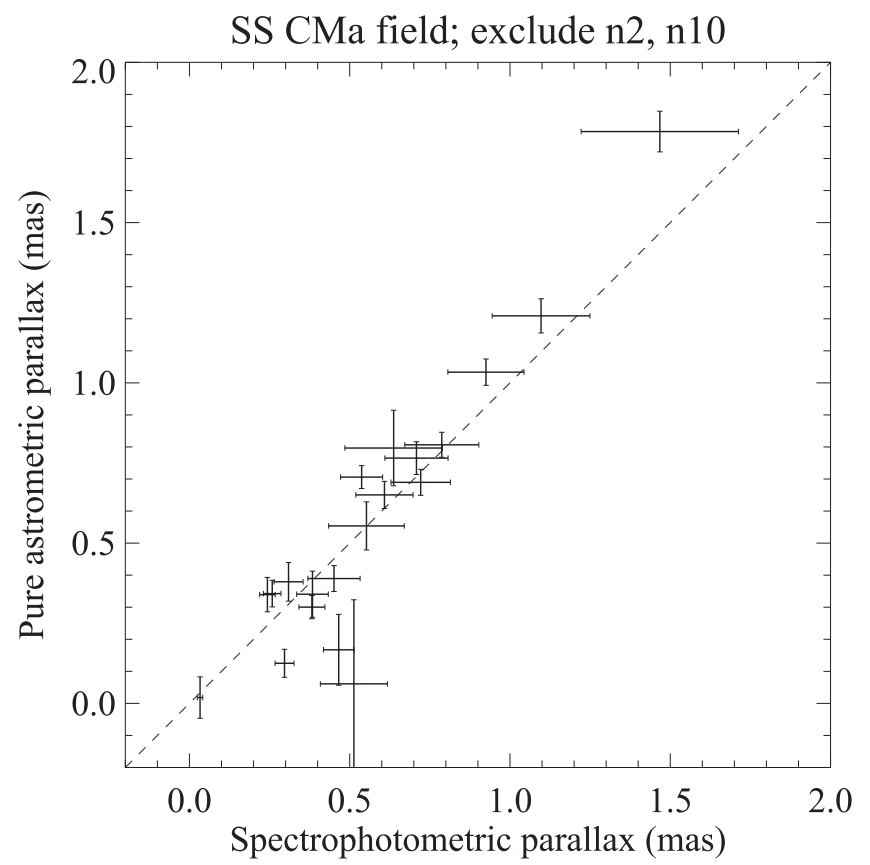

Figure 15. Comparison between spectrophotometric and pure astrometric parallax, obtained by excluding the spectrophotometric prior for each star in turn. Therefore, the pure astrometric parallax for each star is solely based on its astrometric measurements and is not affected by any spectroscopic or photometric data for that star.

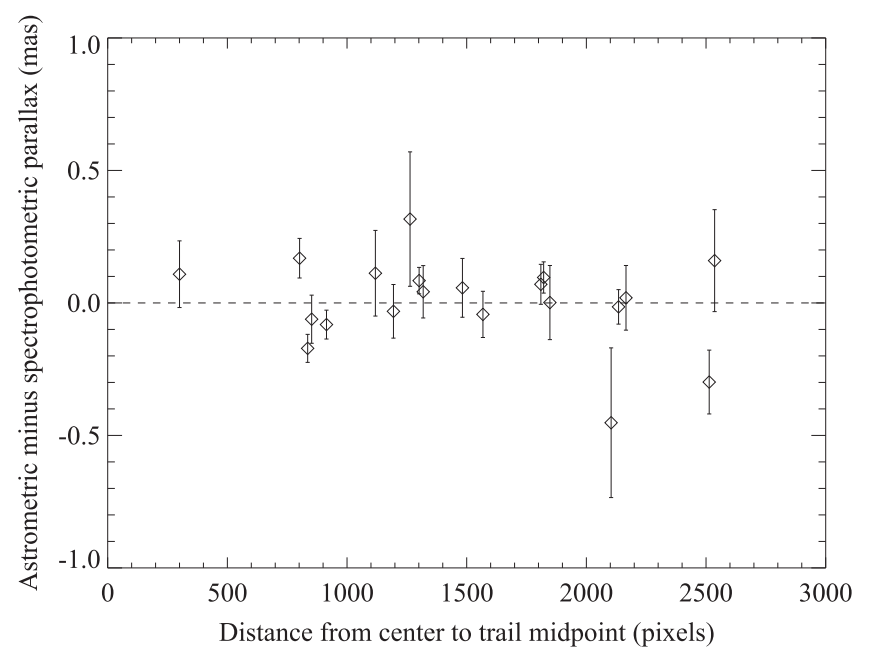

Figure 16. Difference between spectrophotometric and pure astrometric parallax, plotted as a function of the distance of each star from the field center. The error bars combine in quadrature the uncertainties in both parallaxes. The largest deviations occur for stars far from the center of the field, reflecting the difficulty in constraining the polynomial distortion terms when the stars at the edge of the field (which have the most leverage) are excluded from the prior.

a much larger uncertainty than the fully constrained solution. Inspection of the two solutions shows that they differ by about $40 \%$ in the $Y^{2}$ polynomial term, resulting in a differential offset of up to 10 mpix in Epoch 5. The case of Star 10 is even more extreme, with an increase of the nominal error from 0.096 mas to 0.260 mas for the pure astrometric parallax. For most other stars, the uncertainty increases by $10 \%-70 \%$ when the spectroscopic prior is dropped. (Another exception is Star 43, which has a comparatively poor fractional accuracy in the trigonometric parallax because of its very large distance.) Figure 16 shows the difference between pure astrometric and spectrophotometric parallax as a function of distance from the center of the field: two of the three stars with difference larger than 0.3 mas are more than 2000 pixels from the field center.

In summary, we conclude that there is in general good consistency between the astrometric parallaxes we measure from this set of HST spatial scans and the distance estimates obtained from our spectroscopic and multiband photometric measurements. Moreover, as expected, the availability of such estimates for a large fraction of the reference stars is necessary to constrain the overall astrometric solution.

\subsection{Two Special Reference Stars}

Among the reference stars in the field, Star 29 was suggested by Evans \& Udalski (1994) as a possible binary companion to SS CMa, on the basis of its blue color, estimated distance, and likelihood of the relatively small separation of $13^{\prime \prime}$. However, we classify Star 29 as G6 V on the basis of its spectrum (see Table 2), and its estimated spectrophotometric distance modulus is $10.2 \pm 0.3 \mathrm{mag}$, which places it significantly closer than the Cepheid. As shown in Figure 16, the astrometric parallax for Star 29 is in agreement with its spectrophotometric distance, and we conclude that Star 29 is not physically associated with the Cepheid SS CMa, consistent with the upper limit on companion separation found by Evans et al. (2016).

Another interesting reference star is Star 43. Although faint in the visible $\left(V_{606}=15.26 \mathrm{mag}\right)$, this star is quite bright in the near-infrared $(H=10.76 \mathrm{mag})$, and it is classified from the spectrum as a K5 III, with spectrophotometric distance modulus $17.4 \pm 0.5 \mathrm{mag}$. This places the star about $30 \mathrm{kpc}$ from the Sun, well outside the disk and the spheroid of the Galaxy. (Note that this star by itself carries about half the weight of the conversion to absolute parallax for this field.) The trigonometric parallax without spectroscopic prior is $0.018 \pm$ 0.065 mas, hence not significantly detected, but certainly indicative of the star being beyond $10 \mathrm{kpc}$. Such stars are likely rare and are often found through variability studies or special spectral features (see, e.g., the carbon-star selection in Huxor \& Grebel 2015). The Besançon model (Robin \& Crézé 1986; Robin et al. 2003, and references therein) indicates that only 1 in 50 fields at the Galactic coordinates of SS CMa would have a star of comparable brightness beyond $10 \mathrm{kpc}$, and about 1 in 150 beyond $30 \mathrm{kpc}$.

\section{LIMITS ON BINARITY FROM RADIAL VELOCITY OBSERVATIONS}

As mentioned in Section 2.2, there have been suggestions in the literature that SS CMa might be a binary, either from the properties of a nearby star (Star 29 in our list) or from variations of the measured RV. In general, binarity can affect the estimated astrometric parallax if the orbital motion of the Cepheid has a significant component in common with the parallactic motion. Over the short time span of our observations ( $\sim 2$ yr, with 6-month sampling), periods between a few months and $3 \mathrm{yr}$ could have a significant impact on the measured parallax, if the orbital motion is of sufficient amplitude and oriented appropriately. We can rule out that Star 29 is a binary companion on the basis of both its spectrophotometric and astrometric parallax; even if it were physically associated with SS CMa, its impact on the parallax would be negligible owing 
to the extremely long inferred period. On the other hand, a spectroscopic binary companion could in principle impact the astrometric measurement. A priori considerations on the likelihood of binarity as a function of period and mass ratio (see Paper I) suggest that the probability of a significant effect (larger than $10 \mu \mathrm{as}$ ) is $\sim 10 \%$, but these estimates are based primarily on binary statistics obtained for lower-mass stars (see, e.g., Duquennoy \& Mayor 1991), and thus their applicability to massive Cepheids is uncertain. However, in the case of Cepheids, RV measurements can provide useful direct limits on the possibility of binarity and its impact on the measured parallax. A systematic search for binary companions of Cepheids using RV data has been undertaken by Evans et al. (2015), who find a spectroscopic binary fraction of $\sim 30 \%$ for periods between 1 and $20 \mathrm{yr}$ within their detection envelope; SS CMa is not in their sample. We present here an analysis of the RV data we obtained for SS CMa, focusing primarily on the potential impact of a companion on our parallax measurement.

\subsection{Spectroscopic Data}

We observed SS CMa between 2013 April and 2015 November using three different echelle spectrographs: (1) the Hamilton spectrograph (Vogt 1987) at the Shane $3 \mathrm{~m}$ telescope located at Lick Observatory; (2) the Hermes spectrograph (Raskin et al. 2011) at the Flemish $1.2 \mathrm{~m}$ Mercator telescope located at the Roque de los Muchachos Observatory on the island of La Palma, Spain; and (3) the Coralie spectrograph (Queloz et al. 2001) at the Swiss $1.2 \mathrm{~m}$ Euler telescope located at La Silla Observatory, Chile. Data from the Hermes and Coralie spectrographs were reduced using dedicated pipelines. Hamilton spectra were reduced using standard IRAF routines. RVs were determined by crosscorrelation using a numerical mask representative of a solar spectral type (Baranne et al. 1996; Pepe et al. 2002).

RVs from Hermes and Coralie were found to be compatible with each other to within $10-20 \mathrm{~m} \mathrm{~s}^{-1}$, and no zero-point offset was applied. RVs from the Hamilton spectrograph were brought to the Coralie/Hermes zero-point via observations of stable standard stars (HR 4027 and one or more of the following: HD 26161, HR 124, HR 7373, or NSV 7543) using the velocities and zero-point offsets presented by Nidever et al. (2002). Given the uncertainties involved with this zero-point correction, and factoring in the intrinsic precision of the Hamilton RVs, we estimate an uncertainty of approximately $200 \mathrm{~m} \mathrm{~s}^{-1}$ for these data. The individual pipeline-estimated RV uncertainties for Coralie and Hermes range between 20 and $80 \mathrm{~m} \mathrm{~s}^{-1}$. We opt to not include the literature RV data from Joy (1937) and Coulson \& Caldwell (1985) in this analysis, since zero-point offsets $\left(1 \pm 0.5 \mathrm{~km} \mathrm{~s}^{-1}\right.$ for Coulson \& Caldwell 1985) and low precision (typical uncertainties larger than $1 \mathrm{~km} \mathrm{~s}^{-1}$ ) dilute the precision of our new measurements, while not adding significant information for the timescales of interest (1-2 yr).

\subsection{Analysis}

To investigate a possible astrometric signal caused by binarity, we constrain possible values of the projected semimajor axis, $a_{1} \sin i$, for $\mathrm{SS}$ CMa by modeling the RV data. Our model consists of a sum of a 9-harmonic Fourier series, representing the intrinsic velocity variation during the pulsation, and a circular orbital motion. We adopt circular

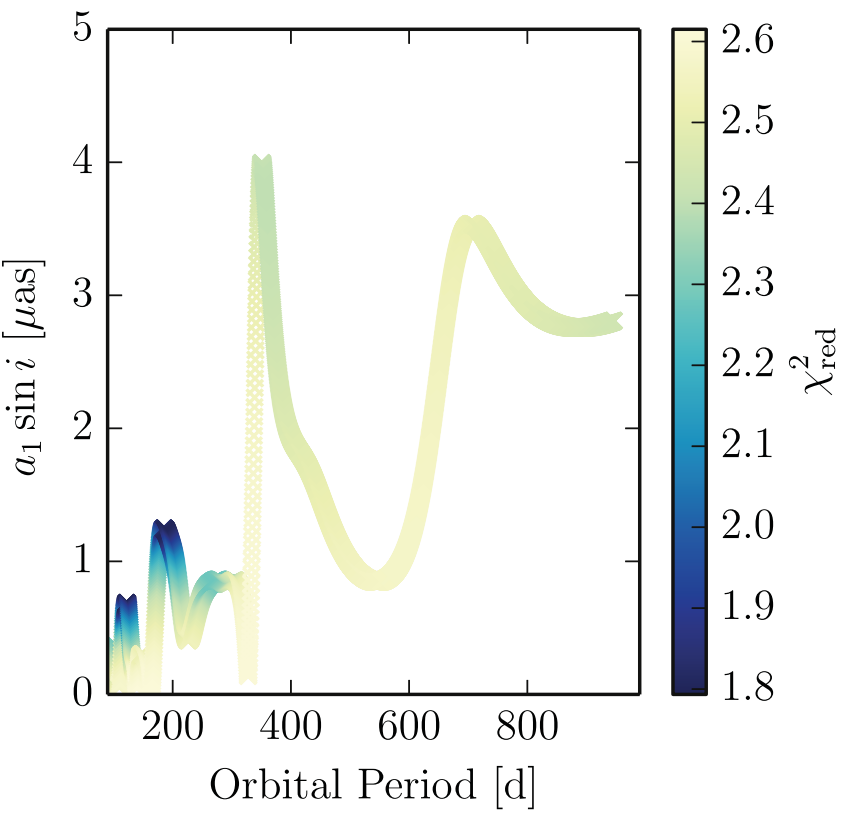

Figure 17. Best-fitting projected semimajor axis as a function of period for the RV data collected for SS CMa. The amplitude shown here assumes that all of the variation in RV is due to orbital motion; in reality, variations in the RV profile during pulsation are likely to contribute substantially to the apparent RV variation, as shown by the fact that the reduced $\chi^{2}$ remains above 1.8 throughout. The color corresponds to the value of the reduced $\chi^{2}$, with darker colors corresponding to better models. The largest possible impact on the astrometric parallax measurement is for an orbital period of $\sim 1 \mathrm{yr}$ and is approximately $\pm 4 \mu \mathrm{as}$; however, a shorter period with a much smaller astrometric impact $(<1.5 \mu \mathrm{as})$ is more consistent with the RV measurements.

orbital motion for simplicity, and since there is no evidence for an eccentric orbit in the available data. We adopt a constant pulsation period of $P=12.3535$ days, which minimizes the scatter in the phased RV data set. We then convert the projected semimajor axis into an astrometric term by assuming a distance of $3 \mathrm{kpc}$. As usual with RV information, this represents a minimum orbital signature, corresponding to an edge-on orbit.

The time sequence of RV measurements indicates a nearly constant systemic RV, excluding even fairly low amplitude deviations (above $\sim 400 \mathrm{~m} \mathrm{~s}^{-1}$ ), consistent with a null detection of orbital motion to within the uncertainty of our measurements. We estimate upper limits on astrometric signals caused by undetected companions, noting that small variations in the pulsation RV pattern already found in several Cepheids (Anderson 2014b) can mimic the effect of low-mass spectroscopic companions (Anderson et al. 2016). Hence, the mere detection of time-variable low-amplitude changes in systemic velocity is not necessarily a clear indication of spectroscopic binarity, as the available sampling of the RV data is insufficient to fully separate these two possibilities. If interpreted as orbital motion, the variation seen in the RV data would result in the astrometric signature shown in Figure 17; darker colors correspond to lower $\chi^{2}$. The maximum impact is around a period of $1 \mathrm{yr}$ with an orbital amplitude of $\sim 4 \mu$ as, while the lowest $\chi^{2}$ occurs for an amplitude of $1.5 \mu \mathrm{as}$. In order to account for the uncertain contribution of the possible orbital motion, we conservatively add a term of $4 \mu$ as in quadrature to the nominal parallax error for SS CMa. Monitoring of this and other Cepheids in our program will continue, and the results will be presented separately in greater detail (R. I. Anderson et al. 2016, in preparation). 


\section{DISCUSSION}

We have presented a trigonometric parallax estimate for SS $\mathrm{CMa}$, the first of 18 Galactic Cepheids in our program of measurements to obtain an improved calibration of the $P-L$ relation of Cepheids with properties comparable to those we are discovering in hosts of SNe Ia within $35 \mathrm{Mpc}$. The parallax of SS CMa is $0.348 \pm 0.038$ mas.

Unlike the pilot case of SY Aur, presented in Paper I, the availability of several bright reference stars and improvements in our analysis procedures result in a formal uncertainty comparable with the pre-observation expectations of 30-40 $\mu$ as. Several of the improvements indicated in Paper I have been implemented for this and all other Cepheids in the sample. For example, we use two shallow and two deep scans at each epoch, we obtain serpentine scans for most epochs, and we have developed and adopted an improved geometric solution that removes a significant fraction of the static ("pattern") difference between observations obtained at different roll angles. We also have been able to quantify and correct for the effect of CTE losses in the detector $X$ direction (X-CTE), although we discovered that the second-epoch observations were not obtained in an optimal position to minimize the X-CTE effect. All of these improvements will be applied to the remaining Cepheids.

In the near term, we expect to complete the five-epoch observations and analysis for the remaining 17 Cepheids in our sample. If we can achieve a comparable accuracy for the other Cepheids, we expect that the overall characterization of the $P-L$ relation exclusively from our parallaxes will yield an uncertainty in the distance-scale calibration of $\sim 2 \%$, providing a powerful and independent test of the present distance scale (Riess et al. 2011, 2016). When combined with improvements in the calibration of the distance of NGC 4258, the contribution of the anchor distance to the calibration of $H_{0}$ is likely to drop to $1.5 \%$. For a subset of nine Cepheids, we will obtain an additional four epochs of observations, which we expect to further reduce the final calibration uncertainty, especially by improving our ability to identify and exclude outliers and learn more about the properties of the telescope.

Additional improvements may result from a simultaneous consideration of the data for all Cepheids. For example, there may be regularities in the long-term behavior of the geometric distortion of the instrument, which we now treat as an unknown term to fit for and subtract. If such regularities prove amenable to a global solution, we may be able to reduce the uncertainty in the final solution by as much as $30 \%$.

In a parallel effort, we are also doubling the sample of nearby SN Ia hosts for which Cepheids are being measured and characterized (Riess et al. 2016; Hoffmann et al. 2016). With this two-pronged approach, we expect to reduce the uncertainty in the local Cepheid-based measurement of $H_{0}$ by $>40 \%$ with respect to Riess et al. (2011). The culmination of the Cepheidbased efforts to refine the local measurement of $H_{0}$ will come with the final results from the Gaia mission (circa 2022), which could permit, when combined with larger samples of SN Ia hosts, a measurement with accuracy of $\sim 1 \%$ or better.

This project was enabled by significant assistance from a wide variety of sources. Merle Reinhardt, George Chapman, William Januszewski, and Ken Sembach provided help with the $H S T$ observations. We thank Ed Nelan, Matt Lallo, Fritz Benedict, and Barbara McArthur for productive discussions about the behavior of the FGS. We also thank Leo Girardi, Alessandro Bressan, and Paola Marigo for the use of and assistance with their Padova isochrone database, Anne Robin for assistance with the Besançon Galaxy Model, Eddie Schlafly and D. Marshall for input on the extinction along the line of sight to SS CMa, and Nolan Walborn for useful discussions on the classification of hot stars.

Support for this work was provided by NASA through programs GO-12879 and GO-13101 from the Space Telescope Science Institute, which is operated by AURA, Inc., under NASA contract NAS 5-26555. A.V.F.'s group at UC Berkeley is also grateful for financial assistance from NSF grant AST1211916, the TABASGO Foundation, and the Christopher R. Redlich Fund. R.I.A. acknowledges funding from the Swiss National Science Foundation as a postdoctoral fellow. S.C. and A.G.R. gratefully acknowledge support by the Munich Institute for Astro- and Particle Physics (MIAPP) of the DFG cluster of excellence "Origin and Structure of the Universe." Research at Lick Observatory is partially supported by a generous gift from Google.

This research is based primarily on observations with the NASA/ESA Hubble Space Telescope, obtained at the Space Telescope Science Institute, which is operated by AURA, Inc., under NASA contract NAS 5-26555. Some of the data presented herein were obtained at the W. M. Keck Observatory, which is operated as a scientific partnership among the California Institute of Technology, the University of California, and the National Aeronautics and Space Administration. The Observatory was made possible by the generous financial support of the W. M. Keck Foundation. Radial velocity measurements are based on observations taken with the Coralie echelle spectrograph mounted to the Swiss $1.2 \mathrm{~m}$ Euler telescope located at La Silla Observatory, Chile, and with the Mercator Telescope, operated on the island of La Palma by the Flemish Community, at the Spanish Observatorio del Roque de los Muchachos of the Instituto de Astrofísica de Canarias. The Euler telescope is supported by the Swiss National Science Foundation. Hermes is supported by the Fund for Scientific Research of Flanders (FWO), Belgium, the Research Council of K. U. Leuven, Belgium, the Fonds National de la Recherche Scientifique (F.R.S.-FNRS), Belgium, the Royal Observatory of Belgium, the Observatoire de Genève, Switzerland, and the Thüringer Landessternwarte, Tautenburg, Germany. We thank all observers who contributed in collecting the ground-based data used in this work, as well as the Euler and Mercator teams for their support.

This publication makes use of data products from the Widefield Infrared Survey Explorer (WISE), which is a joint project of the University of California, Los Angeles, and the Jet Propulsion Laboratory/California Institute of Technology, funded by NASA. It has also made use of the SIMBAD database, operated at CDS, Strasbourg, France.

\section{REFERENCES}

Addison, G. E., Huang, Y., Watts, D. J., et al. 2016, ApJ, 818, 132

Anderson, J. 2014a, The Impact of x-CTE in the WFC3/UVIS Detector on Astrometry, Tech. Rep. (Baltimore, MD: STScI)

Anderson, J., \& Bedin, L. R. 2010, PASP, 122, 1035

Anderson, R. I. 2014b, A\&A, 566, L10

Anderson, R. I., Mérand, A., Kervella, P., et al. 2016, MNRAS, 455, 4231

Baranne, A., Queloz, D., Mayor, M., et al. 1996, A\&AS, 119, 373

Bellini, A., Anderson, J., \& Bedin, L. R. 2011, PASP, 123, 622

Benedict, G. F., McArthur, B. E., Feast, M. W., et al. 2007, AJ, 133, 1810 
Benedict, G. F., McArthur, B. E., Feast, M. W., et al. 2011, AJ, 142, 187 Benedict, G. F., McArthur, B. E., Franz, O. G., et al. 2001, AJ, 121, 1607

Benedict, G. F., McArthur, B. E., Franz, O. G., Wasserman, L. H., \& Henry, T. J. 2000, AJ, 120, 1106

Benedict, G. F., McArthur, B. E., Fredrick, L. W., et al. 2002, AJ, 123, 473 Benedict, G. F., McArthur, B. E., Napiwotzki, R., et al. 2009, AJ, 138, 1969

Bennett, C. L., Larson, D., Weiland, J. L., \& Hinshaw, G. 2014, ApJ, 794, 135

Bennett, C. L., Larson, D., Weiland, J. L., et al. 2013, ApJS, 208, 20

Coulson, I. M., \& Caldwell, J. A. R. 1985, SAAOC, 9, 5

de Bruijne, J. H. J., Rygl, K. L. J., \& Antoja, T. 2015, arXiv:1502.00791

Duquennoy, A., \& Mayor, M. 1991, A\&A, 248, 485

Evans, N. R., Berdnikov, L., Lauer, J., et al. 2015, AJ, 150, 13

Evans, N. R., Bond, H. E., Schaefer, G. H., et al. 2016, arXiv:1603.02224

Evans, N. R., \& Udalski, A. 1994, AJ, 108, 653

Finkbeiner, D. P., Schlegel, D. J., \& Davis, M. 1998, in IAU Coll. 166, The

Local Bubble and Beyond, ed. D. Breitschwerdt, M. J. Freyberg, \& J. Truemper (Berlin: Springer), 367

Francis, C. 2013, MNRAS, 436, 1343

Freedman, W. L., \& Madore, B. F. 2010, ARA\&A, 48, 673

Freedman, W. L., Madore, B. F., Gibson, B. K., et al. 2001, ApJ, 553, 47

Gray, R. O., \& Corbally, C. J. 2014, AJ, 147, 80

Hanson, R. B. 1979, MNRAS, 186, 875

Harrison, T. E., McNamara, B. J., Szkody, P., et al. 1999, ApJL, 515, L93

Hershey, J. L., \& Taff, L. G. 1998, AJ, 116, 1440

Hoffmann, S. L., Macri, L. M., Riess, A. G., et al. 2016, ApJ, submitted

Hoffmeister, C. 1929, AN, 236, 233

Hook, I. M., Jørgensen, I., Allington-Smith, J. R., et al. 2004, PASP, 116, 425

Hu, W. 2005, PhRvD, 71, 047301

Hubble, E. 1929, PNAS, 15, 168

Humphreys, E. M. L., Reid, M. J., Moran, J. M., Greenhill, L. J., \& Argon, A. L. 2013, ApJ, 775, 13

Huxor, A. P., \& Grebel, E. K. 2015, MNRAS, 453, 2653

Joy, A. H. 1937, ApJ, 86, 363

Kodric, M., Riffeser, A., Seitz, S., et al. 2015, ApJ, 799, 144

Leavitt, H. S., \& Pickering, E. C. 1912, HarCi, 173, 1

Livio, M., \& Riess, A. G. 2013, PhT, 66, 41

Lutz, T. E., \& Kelker, D. H. 1973, PASP, 85, 573

MacConnell, D. J., Osborn, W. H., \& Miller, R. J. 1997, AJ, 114, 1268

Marshall, D. J., Robin, A. C., Reylé, C., \& Schultheis, M. 2005, in ESA Special Publication 576, The Three-Dimensional Universe with Gaia, ed. C. Turon, K. S. O'Flaherty, \& M. A. C. Perryman (Noordwijk: ESA), 135

McCarthy, J. K., Cohen, J. G., Butcher, B., et al. 1998, Proc. SPIE, 3355, 81

Michalik, D., Lindegren, L., \& Hobbs, D. 2015, A\&A, 574, A115
Miller, J. S., \& Stone, R. P. S. 1993, The Kast Double Spectrograph, Tech. Rep.

Mora, A., Bastian, U., Biermann, M., et al. 2014, in EAS Publications Ser. 67, The Milky Way Unravelled by Gaia: GREAT Science from the Gaia Data Releases, ed. N. A. Walton et al. (Noordwijk: EAS), 65

Nelan, E. P., \& Bond, H. E. 2013, ApJL, 773, L26

Ngeow, C.-C., Kanbur, S. M., Neilson, H. R., Nanthakumar, A., \& Buonaccorsi, J. 2009, ApJ, 693, 691

Nidever, D. L., Marcy, G. W., Butler, R. P., Fischer, D. A., \& Vogt, S. S. 2002, ApJS, 141, 503

Oosterhoff, P. T. 1935, BHarO, 900, 9

Pepe, F., Mayor, M., Galland, F., et al. 2002, A\&A, 388, 632

Perryman, M. 2009, Astronomical Applications of Astrometry: Ten Years of Exploitation of the Hipparcos Satellite Data (Cambridge: Cambridge Univ. Press)

Planck Collaboration, Ade, P. A. R., Aghanim, N., et al. 2015, arXiv:1502. 01589

Prusti, T. 2012, AN, 333, 453

Queloz, D., Mayor, M., Udry, S., et al. 2001, Msngr, 105, 1

Raskin, G., van Winckel, H., Hensberge, H., et al. 2011, A\&A, 526, A69

Reid, M. J., \& Honma, M. 2014, ARA\&A, 52, 339

Riess, A. G., Casertano, S., Anderson, J., MacKenty, J., \& Filippenko, A. V. 2014, ApJ, 785, 161

Riess, A. G., Macri, L., Casertano, S., et al. 2009, ApJ, 699, 539

Riess, A. G., Macri, L., Casertano, S., et al. 2011, ApJ, 730, 119

Riess, A. G., Macri, L. M., Hoffmann, S. L., et al. 2016, ApJ, in press (arXiv:1604.01424)

Robin, A., \& Crézé, M. 1986, A\&A, 157, 71

Robin, A. C., Reylé, C., Derrière, S., \& Picaud, S. 2003, A\&A, 409, 523

Sandage, A., Tammann, G. A., \& Reindl, B. 2004, A\&A, 424, 43

Sandage, A., Tammann, G. A., Saha, A., et al. 2006, ApJ, 653, 843

Schlafly, E. F., \& Finkbeiner, D. P. 2011, ApJ, 737, 103

Skrutskie, M. F., Cutri, R. M., Stiening, R., et al. 2006, AJ, 131, 1163

Szabados, L. 1996, A\&A, 311, 189

van Altena, W. F., Lee, J. T., \& Hoffleit, E. D. 1995, The General Catalogue of Trigonometric [Stellar] Parallaxes

van Leeuwen, F., Feast, M. W., Whitelock, P. A., \& Laney, C. D. 2007, MNRAS, 379, 723

Vogt, S. S. 1987, PASP, 99, 1214

Wenger, M., Ochsenbein, F., Egret, D., et al. 2000, A\&AS, 143, 9

Wright, E. L., Eisenhardt, P. R. M., Mainzer, A. K., et al. 2010, AJ, 140,1868

Wyman, M., Rudd, D. H., Vanderveld, R. A., \& Hu, W. 2014, PhRvL, 112, 051302 